\title{
ac electrokinetic micropumps: The effect of geometrical confinement, Faradaic current injection, and nonlinear surface capacitance
}

Olesen, Laurits Højgaard; Bruus, Henrik; Ajdari, A.

Published in:

Physical Review E

Link to article, DOI:

10.1103/PhysRevE.73.056313

Publication date:

2006

Document Version

Publisher's PDF, also known as Version of record

Link back to DTU Orbit

Citation (APA):

Olesen, L. H., Bruus, H., \& Ajdari, A. (2006). ac electrokinetic micropumps: The effect of geometrical

confinement, Faradaic current injection, and nonlinear surface capacitance. Physical Review E, 73(5), 056313.

https://doi.org/10.1103/PhysRevE.73.056313

\section{General rights}

Copyright and moral rights for the publications made accessible in the public portal are retained by the authors and/or other copyright owners and it is a condition of accessing publications that users recognise and abide by the legal requirements associated with these rights.

- Users may download and print one copy of any publication from the public portal for the purpose of private study or research.

- You may not further distribute the material or use it for any profit-making activity or commercial gain

- You may freely distribute the URL identifying the publication in the public portal 


\title{
ac electrokinetic micropumps: The effect of geometrical confinement, Faradaic current injection, and nonlinear surface capacitance
}

\author{
Laurits Højgaard Olesen, ${ }^{1,2}$ Henrik Bruus, ${ }^{1}$ and Armand Ajdari ${ }^{2}$ \\ ${ }^{1}$ MIC, Department of Micro and Nanotechnology, Technical University of Denmark, \\ DTU bldg. 345 east, DK-2800 Kongens Lyngby, Denmark \\ ${ }^{2}$ Laboratoire de Physico-Chimie Théorique, UMR CNRS-ESPCI 7083, 10 rue Vauquelin, F-75231 Paris Cedex 05, France
}

(Received 9 October 2005; published 31 May 2006)

\begin{abstract}
Recent experiments have demonstrated that ac electrokinetic micropumps permit integrable, local, and fast pumping (velocities $\sim \mathrm{mm} / \mathrm{s}$ ) with low driving voltage of a few volts only. However, they also displayed many quantitative and qualitative discrepancies with existing theories. We therefore extend the latter theories to account for three experimentally relevant effects: (i) vertical confinement of the pumping channel, (ii) Faradaic currents from electrochemical reactions at the electrodes, and (iii) nonlinear surface capacitance of the Debye layer. We report here that these effects indeed affect the pump performance in a way that we can rationalize by physical arguments.
\end{abstract}

DOI: 10.1103/PhysRevE.73.056313

PACS number(s): 47.65. $-\mathrm{d}, 47.32 .-\mathrm{y}, 85.90 .+\mathrm{h}$

\section{INTRODUCTION}

Lab-on-a-chip systems require micropumps and valves to manipulate small volumes of a liquid sample [1]. Often large external pumps, such as syringe pumps, are used to deliver the necessary pressures, but for portable systems a number of on-chip micropumps have been developed over the last decade. The latter fall into two major categories: One category comprises mechanical actuation and deflectable membranes to create pumps and valves. These have proven to be versatile and simple to fabricate and operate, but there are some difficulties with further downscaling. The second category involves no moving parts but uses electric fields to induce electrokinetic pumping. However, classical dc electroosmosis requires a relatively large voltage and field strength, which is undesirable. Moreover, when a dc current is drawn from electrodes integrated in a microfluidic system there are problems with bubble formation due to electrolysis.

ac electro-osmosis has recently been observed to induce fluid motion over pairs of microelectrodes [2-4]. Based on general symmetry arguments, Ajdari predicted that the same mechanism would generate a net flow over an asymmetric array of electrodes [5], which was soon after demonstrated experimentally by Brown et al. [6] and later by several other groups obtaining pumping velocities $\sim \mathrm{mm} / \mathrm{s}$ with driving voltage of a few volts [7-14].

Theoretically, Ramos et al. performed a more detailed linear response investigation of the pumping mechanism and found a specific pumping direction, a pumping velocity scaling as the square of the driving voltage, and a well-defined frequency for maximal pumping [15].

However, the above analysis is strictly valid only at low driving voltage $V_{0} \ll k_{B} T / e \sim 25 \mathrm{mV}$, whereas experiments are usually performed with at least a few volts. As a consequence, the theory disagrees with experimental observations in several ways: e.g., experimentally the pumping velocity does not always compare well with the predicted $V_{0}^{2}$ scaling, but looks more like a linear scaling $[10,11]$ or tends to saturate at large voltage [13]. Moreover, Studer et al. found a reversal of the pumping direction at driving frequencies well above that for maximal pumping in the "forward" direction [11] and Ramos and co-workers observed reversal of the pumping direction on a traveling-wave device for driving voltages above $2 \mathrm{~V}$ at $1 \mathrm{kHz}$ driving frequency $[13,14]$. The existing theory does not give many clues as to the mechanism for this reversal, except that in the original paper Ajdari predicted pumping in the "reverse" direction at low frequency when Faradaic electrode reactions were included in the model [5]. Indeed, several groups have observed bubble formation and electrode degradation at low frequency and high voltage which indicates that electrode reactions are actually taking place $[8-11,14]$. Lastochkin et al. observed fluid motion in the reverse direction of that usually expected for ac electro-osmosis, which was attributed to strong Faradaic current injection [16]. However, their experiments were performed with driving frequencies in the $\mathrm{MHz}$ range which is of the order of the Debye frequency for the electrolyte, whereas ac electro-osmosis generally occurs around the inverse $R C$ time of the device.

These discrepancies between experimental observations and existing theory demonstrate the need for a more complete theoretical understanding. As a first step we here address a few generalizations of the existing linear response theory to the weakly nonlinear regime by taking into account, within the thin Debye layer approximation, the effect of Faradaic currents both in a linearized scheme and using the full nonlinear Butler-Volmer reaction kinetics. We also include the nonlinear surface capacitance of the Debye layer as described in Gouy-Chapman theory.

The paper is organized as follows: In Sec. II we fix the device geometry and in Sec. III we describe our model for the electrokinetic system. In Sec. IV we extend the linear analysis for low driving voltage to study in more detail the effects of the device geometry and vertical confinement of the system, and we investigate the effect of Faradaic current injection in a linearized scheme. This provides a firm starting point when in Sec. V we study the nonlinear model both with and without Faradaic current. In Sec. VI we summarize our results and compare to experiments reported in the literature, and finally, in Sec. VII we conclude the paper. 


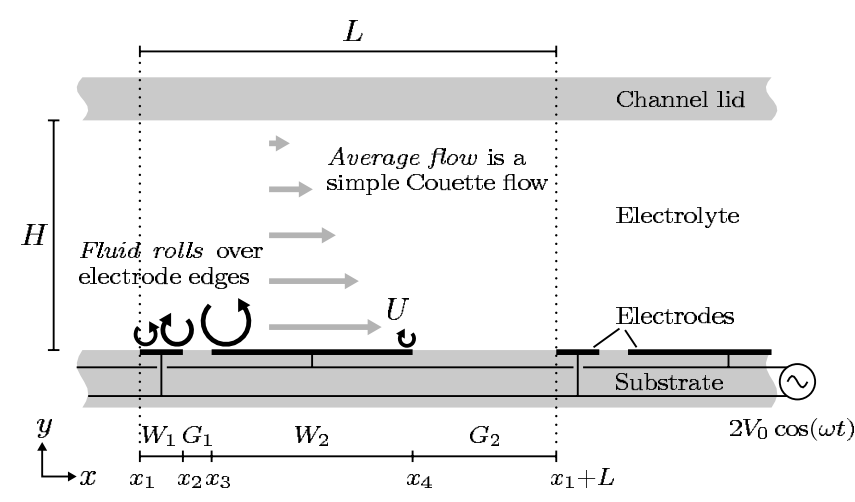

FIG. 1. Sketch of the device geometry. The interdigitated electrode array is biased with an ac voltage that induces a buildup of a Debye screening layer and electro-osmotic fluid motion.

\section{DEVICE GEOMETRY}

We consider a two-dimensional (2D) geometry like that shown in a side view in Fig. 1, similar to devices used experimentally $[6-11,14]$. It consists of a substrate on which an asymmetric array of interdigitated microelectrodes is deposited. Each electrode pair in the array consists of a narrow electrode of width $W_{1}$ and a wide electrode of width $W_{2}$ separated by a narrow and a wide gap $G_{1}$ and $G_{2}$, respectively, and the full period is $L=W_{1}+G_{2}+W_{2}+G_{2}$. Notice that in order to break the left-right symmetry of the array it is necessary that both $W_{1} \neq W_{2}$ and $G_{1} \neq G_{2}$. On top of the electrode array a microfluidic channel of height $H$ is placed and we assume that the device extends sufficiently far into the third dimension that a $2 \mathrm{D}$ description in the $x y$ plane is appropriate.

The channel is filled with an electrolyte, and the narrow and wide electrodes are biased with an ac voltage $V_{\text {ext }}$ $= \pm V_{0} \cos (\omega t)$, respectively; i.e., the applied voltage difference is $2 V_{0} \cos (\omega t)$. This induces the formation of a Debye layer on the electrodes that acts to partially screen the electric field. The nonuniform partial screening gives rise to tangential electric field in the Debye layer and electro-osmotic flow. This flow takes the form of fluid rolls above the electrode edges as indicated in Fig. 1.

\section{ELECTROKINETIC MODEL}

Our model for the electrohydrodynamics of the system is similar to that of Ramos et al. [15] and is based on the following classical approximations [17-20]: (i) The bulk electrolyte is assumed to be charge neutral with uniform salt concentration, such that the ionic transport can be described as Ohmic current. (ii) The Debye layer is assumed to be in local equilibrium with the electrolyte immediately outside the layer, such that the charge distribution and potential variation in the Debye layer can be described by GouyChapman theory. (iii) Moreover, we assume that the thickness of the Debye layer is much smaller than the size of the electrodes, and we neglect surface diffusion and migration of charge. (iv) The bulk fluid motion is described by Stokes flow with a slip condition on the electrodes set by the elec- troosmotic flow induced in the Debye layer. (v) Finally, we also assume that the bulk concentration of the reactants in the Faradaic electrode reaction is constant; i.e., we neglect the effect of mass transfer.

Upon these approximations, the only dynamical variables we are left to consider are the potential distribution $\phi(x, y, t)$ in the bulk and the local surface charge density $q(x, t)$ accumulated in the Debye layer on the electrodes. The instantaneous value of $q$ determines the potential drop from the electrode to the electrolyte immediately outside the Debye layer, whereas the time evolution of $q$ is determined by the balance between the Ohmic current from the bulk and Faradaic current from electrochemical reactions on the electrodes.

We emphasize that this simple electrokinetic model is appropriate only in the weakly nonlinear regime of not-toohigh driving voltage $(\$ 125 \mathrm{mV})$. Beyond this, the assumption of uniform bulk electrolyte concentration breaks down, the effect of mass transfer on the Faradaic reaction kinetics cannot be neglected, and the Debye layer may be driven out of local equilibrium. This is discussed in more detail in Sec. VI.

\section{A. Bulk electrolyte}

In the bulk electrolyte we assume charge neutrality such that the charge continuity equation reduces to

$$
\boldsymbol{\nabla} \cdot \mathbf{J}=0 .
$$

The electric current $\mathbf{J}$ is described simply as the Ohmic current $\mathbf{J}=-\sigma \nabla \phi$ where $\sigma$ is the conductivity of the electrolyte. Since we assume uniform salt concentration and conductivity throughout the bulk, we then simply end up with a Laplace problem for the potential $\phi(\mathbf{r}, t)$.

\section{B. Debye layer}

The Debye layer is assumed to be in local equilibrium with the electrolyte immediately outside the layer. This implies that the driving frequency should be well below the Debye frequency $\omega_{D}=\sigma / \epsilon$ of the electrolyte and that the Faradaic current injection cannot be too strong. The total charge accumulated in the Debye layer can then be directly related to the potential drop $\zeta(x, t)$ across it [18]

$$
\begin{aligned}
q(x, t) & =\int_{\text {Debye layer }} \rho(x, y, t) d y \\
& =-\operatorname{sgn}(\zeta) \sqrt{2 \epsilon k_{B} T \sum_{n} c_{n}^{*}\left(e^{-z_{n} e \zeta / k_{B} T}-1\right)},
\end{aligned}
$$

where $\rho$ is the charge density, $\epsilon$ the permittivity of the solvent, $k_{B}$ Boltzmann's constant, $T$ the temperature, $c_{n}^{*}$ the bulk concentration (number density) of the $n$th ionic species, and $z_{n}$ its valence. In the Debye-Hückel limit $\zeta \ll k_{B} T / z e$, Eq. (3) can be linearized to

$$
q=-\frac{\epsilon}{\lambda_{D}} \zeta,
$$

where $\lambda_{D}=\sqrt{\epsilon k_{B} T / \sum_{n} c_{n}^{*} z_{n}^{2} e^{2}}$ is the Debye length and $\epsilon / \lambda_{D}$ is the Debye layer capacitance (per unit area) at low voltage. 
For larger potentials we focus on the simple case of a monovalent symmetric $(1: 1)$ electrolyte where Eq. (3) reduces to

$$
q=-\frac{\epsilon}{\lambda_{D}} \frac{2 k_{B} T}{e} \sinh \left(\frac{e \zeta}{2 k_{B} T}\right) .
$$

As in the classical Gouy-Chapman-Stern model we assume that the compact (Stern) layer on the electrode simply gives rise to an additional surface capacitance $C_{s}$ in series with the Debye layer [18]. Alternatively $C_{s}$ could model an oxide layer grown intentionally on the electrodes to inhibit electrochemical reactions $[4,12,20]$. In either case we shall for simplicity assume that $C_{s}$ is independent of potential. The total potential drop across the double layer (Debye and Stern) is

$$
V_{\mathrm{ext}}-\phi=\zeta-\frac{1}{C_{s}} q,
$$

where $V_{\text {ext }}$ is the electrode potential and $\phi$ is the potential in the electrolyte immediately outside the Debye layer. The overall capacitance (per unit area) of the double layer is defined as

$$
C(\zeta)=-\frac{q}{V_{\mathrm{ext}}-\phi}
$$

In the Debye-Hückel limit this reduces to

$$
C_{0}=\left[\frac{\lambda_{D}}{\epsilon}+\frac{1}{C_{s}}\right]^{-1}=\frac{1}{1+\delta} \frac{\epsilon}{\lambda_{D}},
$$

where we introduced the parameter $\delta=\epsilon / \lambda_{D} C_{s}$ for the surface capacitance ratio. At larger potential the Debye layer capacitance becomes very large and $C(\zeta)$ is dominated by the Stern layer only. Notice that $C(\zeta)$ always satisfies $C_{0}$ $\leqslant C(\zeta) \leqslant C_{s}$.

The charging of the Debye layer is due to both Ohmic current from the bulk and Faradaic current from electrochemical reactions at the electrode:

$$
\partial_{t} q=-\mathbf{n} \cdot \mathbf{J}+j_{\text {ext }},
$$

where $\mathbf{n}$ is a unit normal pointing out of the electrode, $-\mathbf{n} \cdot \mathbf{J}$ is the Ohmic current running into the Debye layer, and $j_{\text {ext }}$ is the Faradaic current from the electrode reaction. We neglect any surface diffusion and migration of charge in the Debye layer.

\section{Insulating walls}

On the channel substrate between the electrodes and on the channel lid we assume no normal current:

$$
\mathbf{n} \cdot \mathbf{J}=0 \text {. }
$$

This is justified because the amount of charge required to screen any field normal to the channel wall is both very small and builds up on the Debye time scale $\tau_{D}=\epsilon / \sigma$.

\section{Electrochemistry}

For the Faradaic electrode reaction we consider a simple one-step, one-electron redox process of the form

$$
\mathrm{O}+\mathrm{e} \rightleftharpoons \mathrm{R}
$$

where $z_{\mathrm{O}}=z_{\mathrm{R}}+1$ to ensure charge conservation. Activatedcomplex theory predicts an electric current density $j_{\text {ext }}$ from this process given by [18]

$$
j_{\mathrm{ext}}=e\left(k_{\mathrm{R}}^{\ominus} c_{\mathrm{R}}^{s} e^{(1-\alpha) e \Delta \phi_{s} / k_{B} T}-k_{\mathrm{O}}^{\ominus} c_{\mathrm{O}}^{s} e^{-\alpha e \Delta \phi_{s} / k_{B} T}\right),
$$

where $k_{\mathrm{O}}^{\ominus}$ and $k_{\mathrm{R}}^{\ominus}$ are the forward and backward standard rate constants for the reaction, $c_{\mathrm{O}}^{s}$ and $c_{\mathrm{R}}^{s}$ are the concentrations of the oxidized and reduced species directly at the electrode (Stern layer) surface, $\alpha$ is the transfer coefficient, and $\Delta \phi_{s}$ $=-q / C_{s}$ is the potential drop across the Stern layer. Assuming local equilibrium, the concentrations directly at the surface are related to those immediately outside the Debye layer by a Boltzmann factor, $c_{n}^{s}=c_{n} e^{-z_{n} e \zeta / k_{B} T}$. Then Eq. (12) can be reexpressed as the current-overpotential equation

$$
j_{\mathrm{ext}}=j_{0} e^{-\left(z_{\mathrm{O}}-\alpha\right) e \zeta / k_{B} T}\left(\frac{c_{\mathrm{R}}}{c_{\mathrm{R}}^{*}} e^{(1-\alpha) e \vartheta / k_{B} T}-\frac{c_{\mathrm{O}}}{c_{\mathrm{O}}^{*}} e^{-\alpha e \vartheta / k_{B} T}\right),
$$

where $j_{0}=e k^{\ominus}\left(c_{\mathrm{R}}^{*}\right)^{\alpha}\left(c_{\mathrm{O}}^{*}\right)^{1-\alpha}$ is the exchange current, $k^{\ominus}$ is the standard rate constant, $c_{\mathrm{O}}$ and $c_{\mathrm{R}}$ are the concentrations immediately outside the Debye layer, and $c_{\mathrm{O}}^{*}$ and $c_{\mathrm{R}}^{*}$ are the corresponding bulk values. $\vartheta=\left(V_{\text {ext }}-\phi\right)-\left(V_{\text {ext }}-\phi\right)^{\text {eq }}$ is the difference between the actual potential drop across the double layer and the thermal equilibrium for the given redox process and bulk concentration; $\vartheta$ is also termed the overpotential. Appreciating that $c_{n}^{s}$ in Eq. (12) differs from $c_{n}$ by a Boltzmann factor is known as the Frumkin correction to the standard Butler-Volmer equation.

Linearization for small $\vartheta$ and for $c_{\mathrm{O}}$ and $c_{\mathrm{R}}$ close to their bulk values yields

$$
j_{\mathrm{ext}}=j_{0} e^{-\left(z_{\mathrm{O}}-\alpha\right) e \xi^{\mathrm{eq} / k_{B} T}}\left(\frac{c_{\mathrm{R}}}{c_{\mathrm{R}}^{*}}-\frac{c_{\mathrm{O}}}{c_{\mathrm{O}}^{*}}+\frac{e \vartheta}{k_{B} T}\right),
$$

where $\zeta^{\text {eq }}$ is the intrinsic equilibrium potential drop across the Debye layer.

For simplicity we shall take $\zeta^{\mathrm{eq}}=0$ such that $\vartheta=V_{\text {ext }}-\phi$ in Eqs. (13) and (14), set the transfer coefficient $\alpha$ equal to $1 / 2$, and assume that $c_{\mathrm{O}}$ and $c_{\mathrm{R}}$ are virtually at their bulk values; i.e., we neglect the effect of mass transfer. Moreover, for surface potentials outside the Debye-Hückel limit we shall focus on a monovalent symmetric (1:1) electrolyte with $z_{\mathrm{O}}$ $=z_{\mathrm{R}}+1=1$. Then Eqs. (13) and (14) reduce to

$$
j_{\text {ext }}=j_{0} e^{-e \zeta / 2 k_{B} T} 2 \sinh \left[\frac{e\left(V_{\text {ext }}-\phi\right)}{2 k_{B} T}\right]
$$

and

$$
j_{\mathrm{ext}}=j_{0} \frac{e\left(V_{\mathrm{ext}}-\phi\right)}{k_{B} T}=\frac{V_{\mathrm{ext}}-\phi}{R_{\mathrm{ct}}},
$$

respectively, defining the (area specific) charge-transfer resistance $R_{\mathrm{ct}}=k_{B} T / j_{0} e$ of units $\left[\Omega \mathrm{m}^{2}\right]$.

\section{E. Fluid dynamics}

Since we are considering a microsystem where the Reynolds number is usually very low-i.e., where viscosity 
dominates over inertia-the fluid motion in the electrolyte is described by the Stokes equation

$$
\rho_{m} \partial_{t} \mathbf{u}=-\nabla p+\eta \nabla^{2} \mathbf{u},
$$

together with the incompressibility constraint

$$
\boldsymbol{\nabla} \cdot \mathbf{u}=0 .
$$

Here $\rho_{m}$ is the fluid mass density, $\mathbf{u}$ the velocity, $p$ the pressure, and $\eta$ the dynamic viscosity. On the insulating walls the no-slip boundary condition applies, whereas on the electrodes we impose a tangential slip condition based on the Helmholtz-Smoluchowski slip velocity:

$$
u_{s}=-\frac{\epsilon \zeta}{\eta} E_{t},
$$

where $E_{t}=-\partial_{x} \phi$ is the tangential field. This classical result for the slip at a flat surface with an externally applied tangential field also holds in general for thin Debye layers in quasiequilibrium at a metal-electrolyte interface [21].

The fluid flow pattern is complex with rolls above the electrode edges as sketched in Fig. 1. However, we are mostly concerned with the net pumping $\langle Q\rangle=\int_{0}^{H}\left\langle u_{x}\right\rangle d y$. A Fourier analysis shows that on average in time and space the flow is a simple Couette flow driven by the average slip velocity $U$ on the bottom wall [15]

$$
U=\frac{1}{L}\left\{\int_{x_{1}}^{x_{2}}\left\langle u_{s}\right\rangle d x+\int_{x_{3}}^{x_{4}}\left\langle u_{s}\right\rangle d x\right\},
$$

in terms of which $\langle Q\rangle=H U / 2$ - that is, assuming zero backpressure on the device. Here $x_{i}$ denote the positions of the electrode edges (cf. Fig. 1) and the insulating walls do not contribute due to the no-slip condition.

Notice that $u_{s}$ and hence $U$ are determined entirely by the solution to the electrical problem. Therefore we can study pumping without resolving any details of the actual flow pattern above the electrodes-i.e., without solving Eq. (17) — which is very convenient.

\section{F. Characteristic dimensions}

Before going into a detailed analysis it is convenient to discuss the overall properties of the electrokinetic system. Very roughly speaking the system is equivalent to the simple $R C$ circuit shown in Fig. 2. The characteristic length scale $\ell_{0}$ of the device is the narrow electrode gap $G_{1}$, which determines the magnitude of the electric field strength. The characteristic time $\tau_{0}$ is the $R C$ time for charging the Debye layer through the bulk electrolyte $\tau_{0}=R_{0} C_{0}$, where $R_{0}$ is the (area specific) bulk electrolyte resistance. However, at high voltage the Stern layer dominates the capacitance of the double layer and the relaxation time changes to $\tau_{\infty}=R_{0} C_{s}=\tau_{0}(1$ $\left.+\delta^{-1}\right)$. The characteristic time for (de)charging the Debye layer through the Faradaic reaction is $\tau_{\mathrm{ct}}=R_{\mathrm{ct}} C_{0}$. When the electrode reaction is very facile and $R_{\mathrm{ct}} \ll R_{0}$ this can be significantly faster than the Ohmic charging, acting effectively as a "short-circuit" on the Debye layer. Finally, the characteristic fluid velocity obtained with $\zeta \sim V_{0} /(1+\delta)$ and $E_{t}$ $\sim V_{0} / \ell_{0}$ is $u_{0}=\epsilon V_{0}^{2} / \eta \ell_{0}(1+\delta)$.

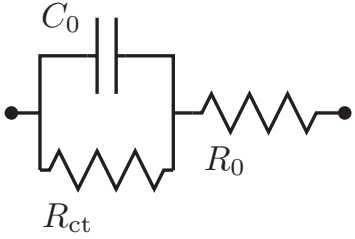

FIG. 2. Equivalent circuit diagram for the electrokinetic system: $R_{0}$ is the bulk electrolyte Ohmic resistance, $C_{0}$ is the double-layer capacitance, and $R_{\mathrm{ct}}$ is the charge-transfer resistance for the electrode reaction. The characteristic time for charging the Debye layer through the electrolyte is $\tau_{0}=R_{0} C_{0}$, and the time scale for decharging the layer through Faradaic electrode reaction is $\tau_{\mathrm{ct}}=R_{\mathrm{ct}} C_{0}$.

\section{LINEAR ANALYSIS}

We first study the problem in the Debye-Hückel limit $\zeta$ $\ll k_{B} T / z e$. Here the electrokinetic problem can be linearized which simplifies the analysis significantly. Indeed the entire problem is characterized by only two dimensionless groups measuring the ratios of the two relaxation times $\tau_{0}$ and $\tau_{\mathrm{ct}}$ and the period of the driving voltage. Of course, the solution also depends on the geometrical parameters $W_{1}, W_{2}, G_{1}, G_{2}$, and $H$, of which four can be varied independently once a characteristic length scale is fixed. We do not vary all these parameters simultaneously, though.

In the simplest case with negligible confinement $(H \gg L)$ and no Faradaic electrode reaction we recover the earlier results of Ramos et al. We also determine the optimal parameters for the geometry of the electrode array. Then with the relative size of the electrodes fixed we consider how vertical confinement of the fluidic channel to $H \lesssim L$ affects the system and we investigate the behavior when electrode reactions occur. Finally, we discuss how part of the linear analysis can be extended to cover systems with arbitrary intrinsic zeta potential $\zeta^{\text {eq }}$ on the electrodes but low driving voltage $V_{0} \ll(1+\delta) k_{B} T / z e$.

\section{A. Dimensionless form for linear analysis}

In preparation of our numerical analysis of the problem we rescale the variables based on the characteristic dimensions defined in Table I, denoting dimensionless variables by a tilde:

$$
\begin{gathered}
\mathbf{r}=\ell_{0} \widetilde{\mathbf{r}}, \quad t=\tau_{0} \widetilde{t}, \quad \omega=\tau_{0}^{-1} \widetilde{\omega}, \\
\phi=V_{0} \widetilde{\phi}, \quad q=C_{0} V_{0} \widetilde{q}, \quad \mathbf{u}=u_{0} \widetilde{\mathbf{u}} .
\end{gathered}
$$

In terms of these, the linearized relations (4), (6), (9), and (16) for the charging of the Debye layer on the electrodes reduce to

$$
\begin{gathered}
\partial_{t} \tilde{q}=\mathbf{n} \cdot \widetilde{\nabla} \widetilde{\phi}+K\left(\widetilde{V}_{\mathrm{ext}}-\tilde{\phi}\right), \\
\tilde{V}_{\mathrm{ext}}-\widetilde{\phi}=-\tilde{q},
\end{gathered}
$$

where $K=R_{0} / R_{\mathrm{ct}}=\tau_{0} / \tau_{\mathrm{ct}}$ is a measure of the facility of the electrode reaction-i.e., a Faradaic conductance. The har- 
TABLE I. Characteristic dimensions of the electrokinetic system and typical experimental values for a $10^{-4} \mathrm{M} \mathrm{KCl}$ working electrolyte, assuming $\delta=0.1$.

\begin{tabular}{lccc}
\hline \hline Geometric length & $\ell_{0}$ & $G_{1}$ & $5 \mu \mathrm{m}$ \\
Debye length & $\lambda_{D}$ & $\sqrt{\epsilon k_{B} T / \Sigma_{n} c_{n}^{*} z_{n}^{2} e^{2}}$ & $30 \mathrm{~nm}$ \\
Ohmic relaxation time & $\tau_{0}$ & $R_{0} C_{0}$ & $70 \mu \mathrm{s}$ \\
at large voltage & $\tau_{\infty}$ & $R_{0} C_{s}$ & $770 \mu \mathrm{s}$ \\
Faradaic relax. time & $\tau_{\mathrm{ct}}$ & $R_{\mathrm{ct}} C_{0}$ & $-\mathrm{s}$ \\
Debye relaxation time & $\tau_{D}$ & $\epsilon / \sigma$ & $0.5 \mu \mathrm{s}$ \\
Double-layer cpt. & $C_{0}$ & $\epsilon / \lambda_{D}(1+\delta)$ & $20 \mathrm{mF} \mathrm{m})^{-2}$ \\
Capacitance ratio & $\delta$ & $\epsilon / \lambda_{D} C_{s}$ & $0.1-$ \\
Bulk resistance & $R_{0}$ & $\ell_{0} / \sigma$ & $3.3 \mathrm{~m} \Omega \mathrm{m}^{2}$ \\
Charge-transfer rst. & $R_{\mathrm{ct}}$ & $k_{B} T / j_{0} e$ & $-\Omega \mathrm{m}^{2}$ \\
Thermal voltage & - & $k_{B} T / e$ & $25 \mathrm{mV}^{-1}$ \\
Fluid velocity & $u_{0}$ & $\epsilon V_{0}^{2} / \eta \ell_{0}(1+\delta)$ & $-\mathrm{ms}^{-1}$ \\
\hline \hline
\end{tabular}

monic time dependence is most conveniently dealt with by introducing complex variables

$$
\widetilde{\phi}(\widetilde{\mathbf{r}}, \widetilde{t})=\frac{1}{2} \hat{\phi}(\widetilde{\mathbf{r}}) e^{i \widetilde{\omega} \widetilde{t}}+\text { c.c. }
$$

and similarly for $\widetilde{V}_{\text {ext }}$ and $\widetilde{q}$. Then $\widetilde{q}$ in Eq. (22) can be eliminated using Eq. (23) to obtain

$$
\mathbf{n} \cdot \widetilde{\nabla} \hat{\phi}=(i \widetilde{\omega}+K)\left(\hat{\phi}-\hat{V}_{\mathrm{ext}}\right)
$$

for the charge balance in the Debye layer. On the insulating walls,

$$
\mathbf{n} \cdot \tilde{\nabla} \hat{\phi}=0,
$$

whereas in the bulk Eq. (1) reduces to a Laplace problem

$$
-\widetilde{\nabla}^{2} \hat{\phi}=0 .
$$

The time average electro-osmotic slip velocity can be expressed as

$$
\left\langle\widetilde{u}_{s}\right\rangle=\left\langle\left(\widetilde{V}_{\mathrm{ext}}-\widetilde{\phi}\right) \partial_{\widetilde{x}} \widetilde{\phi}\right\rangle=-\frac{1}{2} \partial_{\widetilde{x}}\left\langle\left(\widetilde{V}_{\mathrm{ext}}-\widetilde{\phi}\right)^{2}\right\rangle=-\frac{1}{4} \partial_{\widetilde{x}}\left|\hat{V}_{\mathrm{ext}}-\hat{\phi}\right|^{2},
$$

where we used that $\partial_{\widetilde{x}} \widetilde{V}_{\text {ext }}=0$ across the electrodes. The spatial average is then [15]

$$
\begin{aligned}
\widetilde{U}= & \frac{1}{\widetilde{L}}\left\{\int_{\widetilde{x}_{1}}^{\tilde{x}_{2}}\left\langle\widetilde{u}_{s}\right\rangle d \widetilde{x}+\int_{\widetilde{x}_{3}}^{\tilde{x}_{4}}\left\langle\widetilde{u}_{s}\right\rangle d \widetilde{x}\right\} \\
= & -\frac{1}{4 \widetilde{L}}\left(\left|\hat{V}_{\mathrm{ext}}-\hat{\phi}\right|_{\tilde{x}_{2}}^{2}-\left|\hat{V}_{\mathrm{ext}}-\hat{\phi}\right|_{\tilde{x}_{1}}^{2}\right. \\
& \left.+\left|\hat{V}_{\mathrm{ext}}-\hat{\phi}\right|_{\tilde{x}_{3}}^{2}-\left|\hat{V}_{\mathrm{ext}}-\hat{\phi}\right|_{\tilde{x}_{4}}^{2}\right) .
\end{aligned}
$$

\section{B. Validation of the numerical scheme}

We solve Eqs. (25)-(27) numerically with the finiteelement method, using the commercial software package

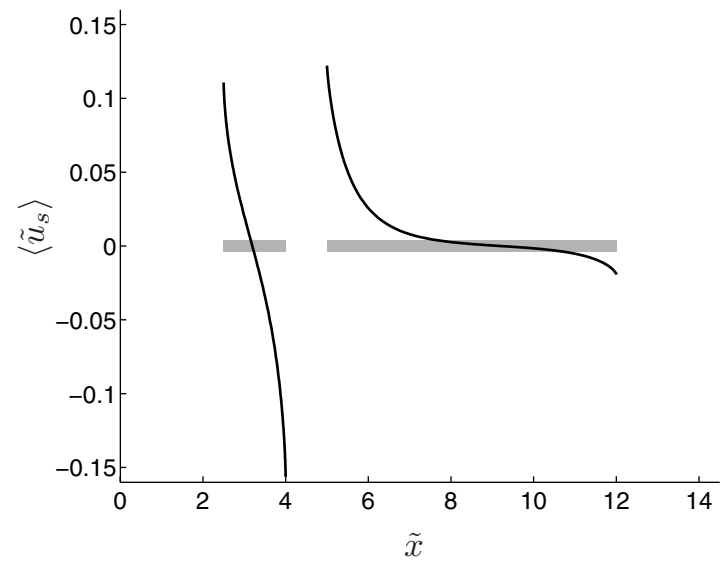

FIG. 3. Time average slip velocity $\left\langle\tilde{u}_{s}\right\rangle$ across the electrodes obtained for $\widetilde{\omega}=1$ and $K=0$ (no Faradaic current) in a geometry with $\widetilde{W}_{1}=1.5, \widetilde{W}_{2}=7, \widetilde{G}_{2}=5$, and $\widetilde{H} \gg \widetilde{L}$.

FEMLAB. We use second-order Lagrange elements to approximate the solution and a nonuniform finite-element mesh with increased resolution at the electrode edges.

In the simple case without any Faradaic current, the Debye layer is charged by Ohmic current through the bulk only. Figure 3 shows the time average slip velocity $\left\langle\widetilde{u}_{s}\right\rangle$ across the electrodes for $\widetilde{\omega}=1$ and $K=0$ in a geometry with $\widetilde{W}_{1}=1.5$, $\widetilde{W}_{2}=7, \widetilde{G}_{2}=5$, and $\widetilde{H} \gg \widetilde{L}$. The slip velocity is strongest close to the edges of the electrodes and is always directed from the edges towards the center of the electrodes. To understand this behavior, notice that close to the electrode edges the electric field and hence the Ohmic current are stronger, which makes the charging of the Debye layer faster and the screening of $\widetilde{V}_{\text {ext }}$ more efficient. Then Eq. (28) clearly shows that $\left\langle\widetilde{u}_{s}\right\rangle$ is always directed from regions where the screening is good and $\left|\hat{V}_{\text {ext }}-\hat{\phi}\right|^{2}$ is large towards regions where the screening is less efficient and $\left|\hat{V}_{\text {ext }}-\hat{\phi}\right|^{2}$ is smaller-i.e., from the electrode edges towards the center.

The slip velocity on the electrodes gives rise to bulk fluid motion with rolls above the electrode edges as sketched in Fig. 1. However, the net pumping is determined only by the average slip $\tilde{U}$ which is shown in Fig. 4 as a function of frequency $\widetilde{\omega}$. When $\widetilde{\omega} \ll 1$ the screening is almost complete at all times. Hence, there is no tangential field and no electroosmotic flow. Conversely, when $\widetilde{\omega} \gg 1$ the driving is too fast for any significant screening to occur; i.e., there is no charge in the Debye layer and no flow. As a consequence, in Fig. 4 we see that the pumping velocity $\widetilde{U}$ is maximized around $\widetilde{\omega}$ $\sim 1$, whereas it falls off as $\widetilde{\omega}^{2}$ at low frequency and slightly faster than $\widetilde{\omega}^{-2}$ at high frequency.

In the low-frequency limit $\widetilde{\omega} \ll 1$ we can verify our numerical results analytically by expanding the problem in powers of $\widetilde{\omega}[22]$. It then turns out that the leading-order time average slip $\left\langle\widetilde{u}_{s}\right\rangle$ scales as $\widetilde{\omega}^{2}$ with a corresponding pumping velocity $\widetilde{U}$ shown with a dashed line in Fig. 4 . It is interesting to note that the overall magnitude of $\tilde{u}_{s}$ falls off only linearly with $\widetilde{\omega}$ at low frequency. This results from the uniform surface charge density to zeroth order in $\widetilde{\omega}$ interacting 


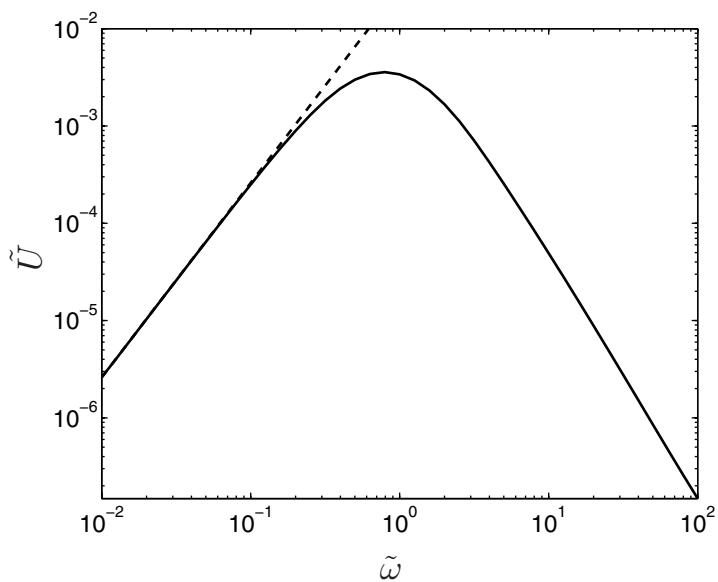

FIG. 4. Pumping velocity $\widetilde{U}$ as a function of frequency $\widetilde{\omega}$. Dashed line: analytical result at low frequency where $\widetilde{U} \propto \widetilde{\omega}^{2}$.

with the first-order electric field, which gives rise to oscillating flow but no time average. A similar expansion in the high-frequency limit is more difficult. Still, our numerical results show that the magnitude of $\widetilde{u}_{s}$ falls off as $\widetilde{\omega}^{-2}$ at high frequency for fixed $\tilde{x}$, but also that it remains finite in a narrow region of width $\widetilde{\omega}^{-1}$ from the electrode edges. The latter appears to be an artifact due to our simplified geometry model with infinitely thin electrodes: For electrodes of finite thickness and radius of curvature $\widetilde{R}$ at the edges, the magnitude of $\widetilde{u}_{s}$ falls of as $\widetilde{\omega}^{-2}$ everywhere on the electrodes for $\widetilde{\omega} \gg \widetilde{R}^{-1}$; see also [23].

One particular question that one might ask from Fig. 4 is why the maximum net pumping velocity $\widetilde{U}_{\max } \sim 0.003$ is so low: If the chosen velocity scale $u_{0}$ is appropriate, should we not expect $\widetilde{U}_{\max } \sim 1$ ? Starting from Fig. 3 we note that already the time average slip $\left\langle\widetilde{u}_{s}\right\rangle$ is less than $O(1)$ because the double-layer potential drop $\left|\hat{V}_{\text {ext }}-\hat{\phi}\right|$ in Eq. (28) is only around half the total applied voltage $\hat{V}_{\text {ext }}$ at the relaxation frequency. The spatial average over the full period of the array includes both positive and negative values of $\left\langle\widetilde{u}_{s}\right\rangle$ and passive sections of insulating channel wall, which reduces the value of $\tilde{U}$ further compared to unity. Interestingly, the average slip across only the narrow electrode,

$$
\tilde{U}_{1}=\frac{1}{\widetilde{L}} \int_{\widetilde{x}_{1}}^{\tilde{x}_{2}}\left\langle\widetilde{u}_{s}\right\rangle d \widetilde{x}
$$

changes sign for $\widetilde{\omega} \sim 1$. At low frequency, $\widetilde{U}_{1}$ and the corresponding average $\widetilde{U}_{2}$ across the wide electrode are both positive with $\widetilde{U}_{1} \sim 0.1 \widetilde{U}_{2} \propto \widetilde{\omega}^{2}$. Above the relaxation frequency we have $-\widetilde{U}_{1} \sim \widetilde{U}_{2} \propto \omega^{-1}$, although their sum $\widetilde{U}=\widetilde{U}_{1}+\widetilde{U}_{2}$ falls off as (or slightly faster than) $\widetilde{\omega}^{-2}$.

In order to further validate our numerical scheme we compare to the results obtained by Ramos et al. using a boundary element method based on the Greens function for a unit charge line source [15]. This is shown in Fig. 5, where the time average slip $\left\langle\widetilde{u}_{s}\right\rangle$ from both electrodes is shown on

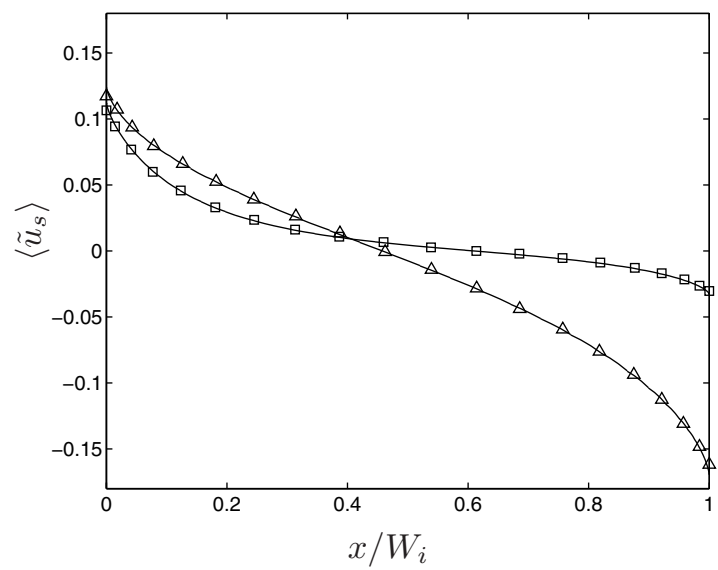

FIG. 5. Time average slip velocity $\left\langle\tilde{u}_{s}\right\rangle$ for both electrodes against the relative position on the electrode $x / W_{i}$. Symbols: results of Ramos et al. [15] for the narrow $(\triangle)$ and wide $(\square)$ electrodes, respectively. Solid lines: our numerical solution.

the same graph for $\widetilde{\omega}=1.24$ in a geometry with $\widetilde{W}_{1}=1, \widetilde{W}_{2}$ $=\widetilde{G}_{2}=10 / 3$, and $\widetilde{H} \gg \widetilde{L}$. The results from the two different numerical schemes are hardly distinguishable, except close to the electrode edges where our finite-element solution displays a slightly larger slip velocity [24].

We also checked our results for convergence with mesh refinement. The potential $\hat{\phi}(\mathbf{r})$ is everywhere smooth, but the tangential field is singular at the electrode edges due to the abrupt change in boundary condition between Eqs. (25) and (26) [25]. Of course, our finite-element solution cannot represent an infinite slope, but by choosing a strongly nonuniform mesh with very fine resolution near the edges we obtain fairly good quality of the solution even close to the singularity. Moreover, we are mostly concerned with the net pumping $\widetilde{U}$ which is an integral quantity and therefore less sensitive to the details at the electrode edges [26].

\section{Optimal device geometry}

An important question in relation to actual device design is what electrode geometry would maximize the pumping velocity. It is immediately clear that since the characteristic velocity $u_{0}$ is inversely proportional to the characteristic length scale $\ell_{0}$, the pumping is increased by downscaling the overall size of the electrodes. We focus again on the limit $H \gg L$, where the results are independent of $H$. Still, there remains four different length scales describing the electrode geometry-namely, $W_{1}, W_{2}, G_{1}$, and $G_{2}$ - of which three can be varied independently once a characteristic length scale is fixed.

Ramos et al. also investigated how the pumping velocity depends on the electrode geometry [15]. They rescaled the problem using the width of the wide electrode $W_{2}$ as their characteristic length scale, but found that the pumping velocity would increase without bounds as $G_{1} / W_{2} \rightarrow 0$. Therefore they fixed the ratio $G_{1} / W_{2}$ at 0.1751 , as in the experiment by Brown et al. [6], and found that the pumping velocity was then maximized when $W_{1} / W_{2}=0.24$ and $G_{2} / W_{2}=0.8$. 
TABLE II. List of geometries used experimentally and our linear model prediction for their dimensionless maximum pumping velocity and optimal driving frequency. Of course, the actual pumping velocity depends on the absolute size of the device through $u_{0}$.

\begin{tabular}{llllllc}
\hline \hline & $\tilde{W}_{1}$ & $\tilde{W}_{2}$ & $\tilde{G}_{2}$ & $\tilde{U}_{\max }$ & $\widetilde{\omega}_{\max }$ & Refs. \\
\hline Brown et al. & 0.93 & 5.7 & 3.5 & 0.00331 & 1.12 & {$[6,7,11]$} \\
Studer et al. & 0.67 & 4.33 & 2.67 & 0.00293 & 1.47 & {$[8]$} \\
Mpholo et al. & 1.0 & 5.0 & 3.0 & 0.00332 & 1.12 & {$[9]$} \\
Debesset et al. & 0.8 & 5.2 & 3.2 & 0.00317 & 1.26 & {$[10]$} \\
Ramos et al. & 1.37 & 5.71 & 4.57 & 0.00357 & 0.88 & {$[15]$ (theory) } \\
Global optimum & 1.51 & 6.55 & 4.74 & 0.00359 & 0.80 & Present work \\
\hline \hline
\end{tabular}

However, the choice $G_{1} / W_{2}=0.1751$ is arbitrary. It is more appropriate to identify the narrow electrode gap $G_{1}$ as the characteristic length scale and look for an optimal device geometry as a function of $\widetilde{W}_{1}, \widetilde{W}_{2}$, and $\widetilde{G}_{2}$ in the parameter ranges $0<\widetilde{W}_{1} \leqslant \widetilde{W}_{2}<\infty$ and $1 \leqslant \widetilde{G}_{2}<\infty$. Notice that when $\widetilde{W}_{2}$ and $\widetilde{G}_{2}$ attain their lower limits, the net pumping $\widetilde{U}$ vanishes due to left-right symmetry; i.e., the optimum cannot be on the parameter range boundaries. Therefore we can use a simple unconstrained optimization algorithm such as the MATLAB function FMINSEARCH to determine the global optimum:

$$
\tilde{U}_{\max }=\max _{\left[\widetilde{\omega}, \tilde{W}_{1}, \tilde{W}_{2}, \tilde{G}_{2}\right]}\left\{\tilde{U}\left(\widetilde{\omega}, K=0, \tilde{W}_{1}, \tilde{W}_{2}, \widetilde{G}_{2}\right)\right\} .
$$

In this way we find that the pumping velocity is maximized for $\tilde{W}_{1}=1.51, \quad \tilde{W}_{2}=6.55, \quad$ and $\tilde{G}_{2}=4.74$, where $\tilde{U}_{\max }$ $=0.00359$ at $\widetilde{\omega}_{\max }=0.80$.

Given that we have tracked down the global optimum, it is natural to ask how much better this geometry performs as compared to that suggested by Ramos et al. and those used experimentally. This is summarized in Table II: Almost all experimental geometries come within $10 \%$ of the maximal pumping velocity, and the theoretical result of Ramos et al. is less than $1 \%$ off. Yet the frequency $\widetilde{\omega}_{\max }$ at which pumping is maximized for the various geometries differs by almost a factor of 2 .

The overall conclusion to be drawn is that the pumping velocity is fairly insensitive to the particular choice of electrode geometry. Henceforth, we shall therefore continue to use a geometry with rounded numbers $\widetilde{W}_{1}=1.5, \widetilde{W}_{2}=7$, and $\widetilde{G}_{2}=5$ which is still close to optimal; see also Fig. 6 .

\section{Confined geometry}

In several experimental studies the height of the pumping channel $H$ has been comparable to the period of the electrode array $L[8,10,11]$. It is not immediately clear how this would affect the properties of the device compared to the simple case of no confinement $H \gg L$.

Figure 7 shows the pumping velocity $\tilde{U}$ as a function of frequency $\widetilde{\omega}$ and degree of confinement $L / H$. Our previous results correspond to the base line $L / H \ll 1$, whereas the confinement becomes significant for $L / H \gtrsim 2$. In particular, the pumping velocity increases by roughly a factor of 2 and the optimal driving frequency $\widetilde{\omega}_{\max }$ becomes proportional to $\tilde{H}$ for $L / H \gg 1$. The latter is easily understood from our simple circuit model of the system in Fig. 2: While the double-layer capacitance $C_{0}$ is largely independent of confinement, the resistance of the bulk electrolyte changes from $R_{0}$ to $R_{0} / \tilde{H}$ because the cross section of the conducting channel is decreased. The relaxation frequency therefore becomes $\tilde{H} / R_{0} C_{0}$-i.e., proportional to $\tilde{H}$.

Our findings here are important in relation to actual device design because they indicate that working with a strongly confined geometry does not, as one might have worried, mask the asymmetry and destroy the ability to pump. Thus the channel height $H$ and the period of the electrode array $L$ can be chosen independently. It is important to realize that while the pumping velocity $U$ does not depend much on confinement, the maximal flow rate $Q_{\max }=H U / 2$ that the pump can deliver (per unit width of the channel) and the maximal backpressure $\Delta p_{\max }=6 \eta N L U / H^{2}$ it can sustain (where $N$ is the total number of electrode pairs) certainly do.

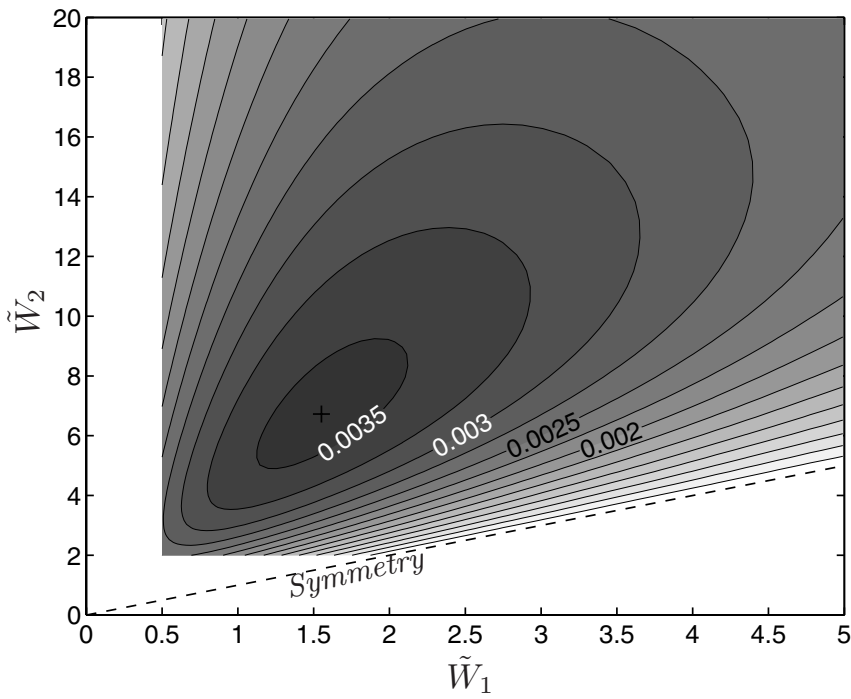

FIG. 6. Contour plot of the maximal pumping velocity over frequency $\max _{\widetilde{\omega}}\{\widetilde{U}(\widetilde{\omega}, \ldots)\}$ as a function of $\widetilde{W}_{1}$ and $\widetilde{W}_{2}$ for $\widetilde{G}_{2}=5$. When $\widetilde{W}_{1}=\tilde{W}_{2}$ the net pumping vanishes due to symmetry. There is a unique optimum close to $\widetilde{W}_{1}=1.5$ and $\tilde{W}_{2}=7$ with $\tilde{U}_{\max }$ $=0.00358$ and $\widetilde{\omega}_{\max }=0.8$. 


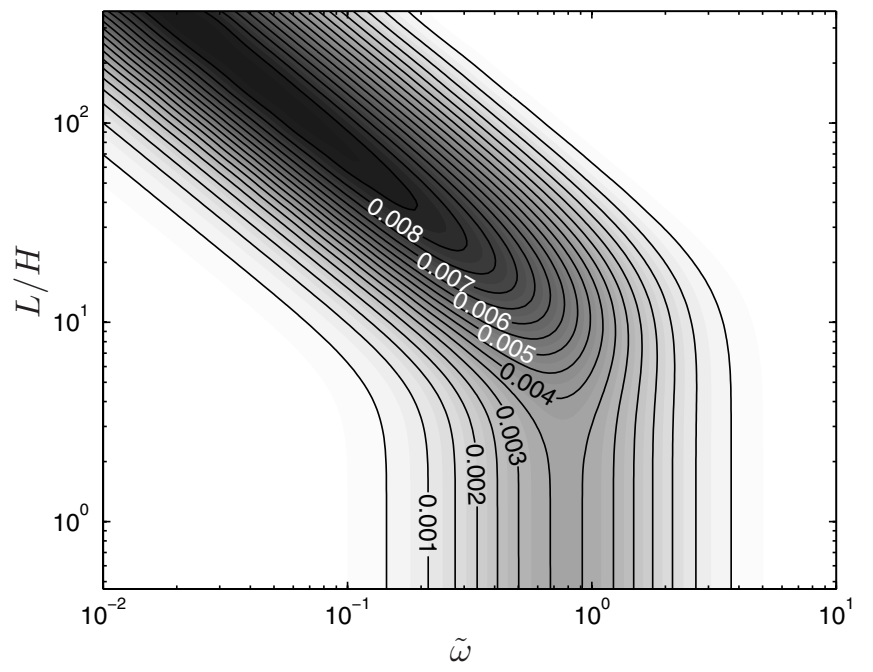

FIG. 7. Contour plot of pumping velocity $\tilde{U}$ as a function of frequency $\widetilde{\omega}$ and degree of confinement $L / H$.

\section{E. Faradaic current injection}

When we include electrode reactions and Faradaic current in our model, one of the most important new features is that the complete screening of the electrodes at low frequency is unbalanced. When the reaction is slow and the Faradaic conductance $K=R_{0} / R_{\mathrm{ct}} \ll \tilde{\omega}$, the effect is small and only perturb the result from purely Ohmic charging slightly. Conversely, when $K \gg \widetilde{\omega}$ the reaction is fast enough to establish a steady state where the Ohmic and Faradaic currents are balancing. For either $K \gg 1$ and/or $\widetilde{\omega} \gg 1$ the Debye layer is effectively "short-circuited;" cf. Fig. 2. On the basis of these observations we would expect to see pumping even in the lowfrequency limit when the Faradaic reaction speed is moderate and $K \sim 1$.

Figure 8 shows the pumping velocity $\tilde{U}$ as a function of $\widetilde{\omega}$ and $K$. For $K \ll \tilde{\omega}$ we recover our previous results for pure

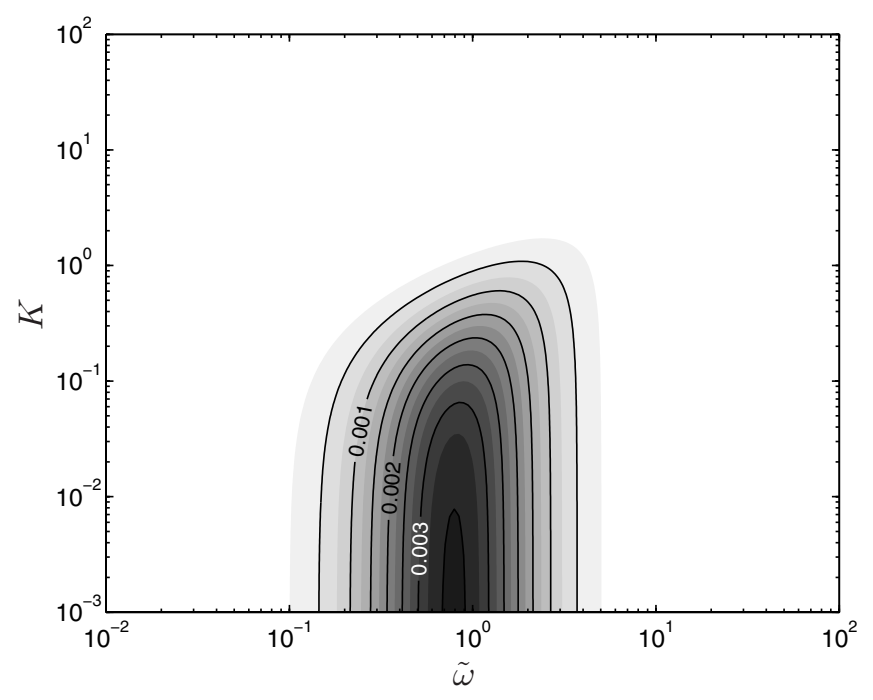

FIG. 8. Contour plot of the pumping velocity $\widetilde{U}$ as a function of frequency $\widetilde{\omega}$ and Faradaic conductance $K$.

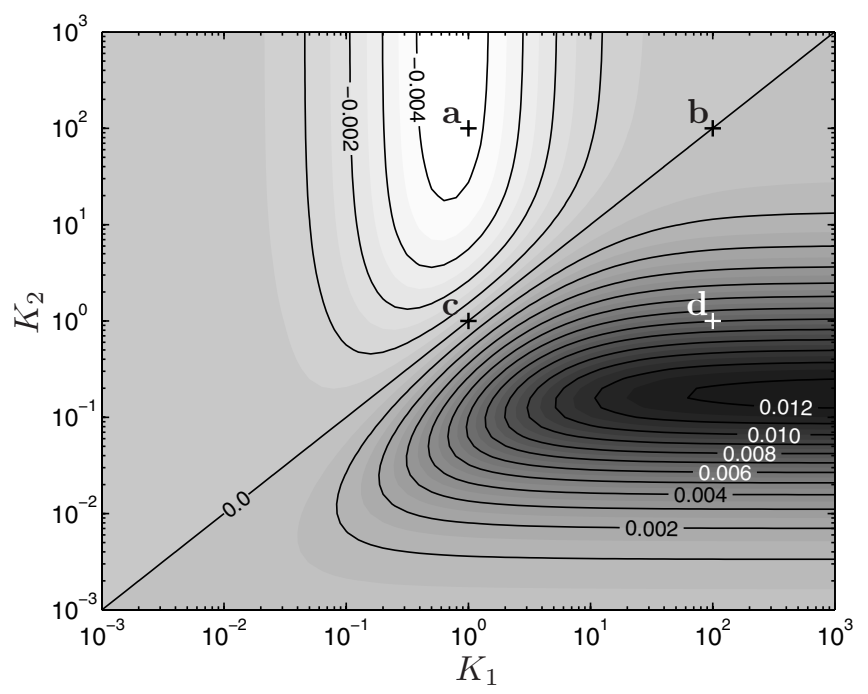

FIG. 9. Contour plot of $\tilde{U}$ in the zero-frequency limit $\tilde{\omega} \sim 0$ with asymmetric current injection: $K_{1}$ and $K_{2}$ denote the Faradaic conductance on the narrow and wide electrodes, respectively. The slip velocity $\left\langle\widetilde{u}_{s}\right\rangle$ at the points (a)-(d) is shown in Fig. 10.

Ohmic charging. However, for $K \gg \widetilde{\omega}$ we observe no pumping at all, not even for $K \sim 1$. Figure 9 shows the result of a more detailed investigation in the zero-frequency limit $\widetilde{\omega}$ $\sim 0$, where we consider a device with asymmetric surface properties on the electrodes, such that the Faradaic conductance takes the values $K_{1}$ and $K_{2}$ on the narrow and wide electrodes, respectively. The figure shows that whenever $K_{1}$ $\neq K_{2}$ the device does actually pump: When $K_{1}>K_{2}$ it pumps in the forward direction-i.e., in the same direction as observed for purely Ohmic charging-but for $K_{2}>K_{1}$ the pumping direction is reversed.

This reversal of the pumping direction can be understood qualitatively by inspecting the form of the time average slip $\left\langle\tilde{u}_{s}\right\rangle$ for the four different points marked (a) to (d) in Fig. 9, the result of which is shown in Fig. 10. When $K_{1}=K_{2}=1$ (c) the slip velocity is clearly nonzero on both electrodes, but the average across the narrow electrode exactly cancels that across the wide electrode. When $K_{1} \gg K_{2}=1$ (d) the Debye layer on the narrow electrode is essentially short-circuited by the fast electrode reaction, leaving no charge and no electroosmotic flow there except from in a narrow region of width $\sim K_{1}^{-1}$ from the electrode edges. Therefore the pumping velocity $\tilde{U}$ is dominated by the contribution from the wide electrode. Similarly, when $K_{2} \gg K_{1}=1$ (a) the Debye layer on the wide electrode is essentially short-circuited and the pumping velocity is dominated by the contribution from the narrow electrode-which tends to be negative.

In conclusion, the absence of pumping in the lowfrequency limit when $K_{1}=K_{2}$ is a special case due to high symmetry; whenever $K_{1} \neq K_{2}$ there is some pumping and this falls off linearly with $K_{i}$ for $K_{i} \ll 1$. More generally we can prove that for a device with spatially varying capacitance ratio $\delta(\widetilde{x})$ and Faradaic conductance $K(\widetilde{x})$, there can be no pumping in the low-frequency limit when the product [1 $+\delta(\tilde{x})] K(\widetilde{x})$ is a constant on all electrodes. The details of the proof are outlined in Appendix A. 

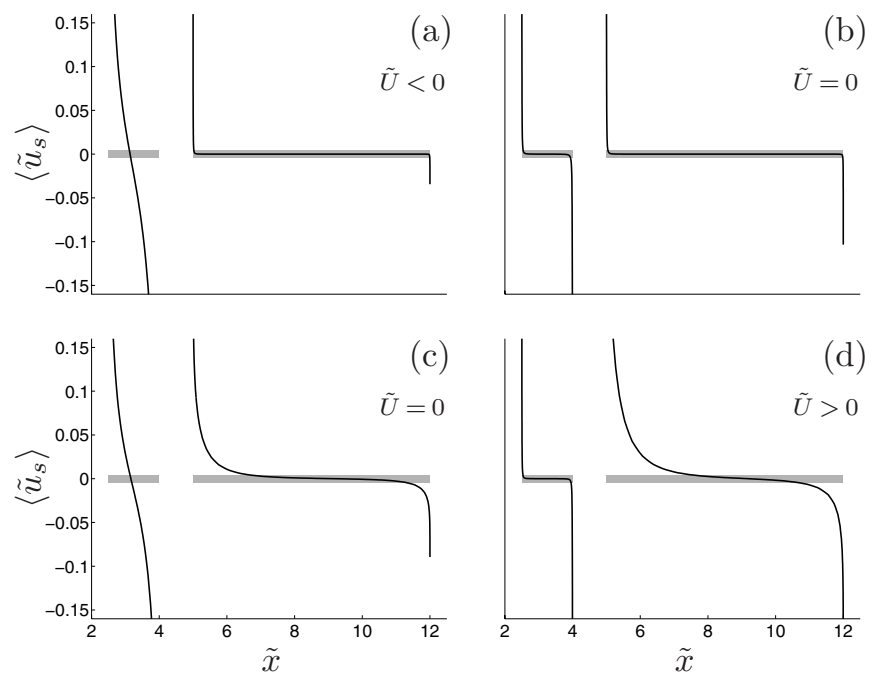

FIG. 10. Time average slip velocity $\left\langle\tilde{u}_{s}\right\rangle$ on the electrodes for the parameter values marked in Fig. 9: (a) $K_{1}=1, K_{2}=10^{2}$; (b) $K_{1}$ $=K_{2}=10^{2}$; (c) $K_{1}=K_{2}=1$; (d) $K_{1}=10^{2}, K_{2}=1$.

\section{F. Linear response at nonzero intrinsic zeta potential}

Many electrode-electrolyte systems spontaneously form a Debye layer and possess an intrinsic zeta potential typically of the order $\zeta^{\text {eq }} \sim 100 \mathrm{mV}$ which is well beyond the DebyeHückel limit $\zeta \ll k_{B} T / z e \sim 25 \mathrm{mV}$. Hence, our foregoing linear analysis cannot be applied immediately to such systems. But then it is standard procedure in electrochemical impedance spectroscopy to study the linear response of the electrokinetic system to a small applied voltage [18].

Consider a system where the electrolyte forms a Debye layer on the electrodes with an intrinsic zeta potential $\zeta^{\text {eq }}$. In equilibrium there is a uniform surface charge density $q^{\text {eq }}$ given by Eq. (3) on the electrodes and a constant potential $\phi^{\mathrm{eq}}=-\zeta^{\mathrm{eq}}+q^{\mathrm{eq}} / C_{s}$ in the bulk. If the electrode array is biased with a small-amplitude ac voltage $V_{\text {ext }}= \pm V_{0} \cos (\omega t)$, we can linearize around equilibrium and writing $\phi=\frac{1}{2}\left(\phi^{\mathrm{eq}}+\hat{\phi}^{\prime} e^{i \omega t}\right)$ + c.c. and similarly for $q$ and $\zeta$ we recover the form of Eqs. (26) and (27) for the linear response $\hat{\phi}^{\prime}$. Only now the characteristic time used for the rescaling in Eq. (21) becomes

$$
\tau_{0}=R_{0} C_{d}
$$

where $C_{d}\left(\zeta^{\mathrm{eq}}\right)$ is the differential capacitance of the double layer:

$$
C_{d}=-\frac{d q}{d\left(V_{\mathrm{ext}}-\phi\right)} .
$$

For a symmetric $z: z$ electrolyte this can be written as

$$
C_{d}=\frac{\epsilon}{\lambda_{D}\left[\delta+\operatorname{sech}\left(z e \zeta^{\mathrm{eq}} / 2 k_{B} T\right)\right]} .
$$

The relaxation time for the Faradaic electrode reaction becomes

$$
\tau_{\mathrm{ct}}=R_{\mathrm{ct}} C_{d}
$$

where $R_{\mathrm{ct}}\left(\zeta^{\mathrm{eq}}\right)$ is the differential charge-transfer resistance given by

$$
\frac{1}{R_{\mathrm{ct}}}=\frac{d j_{\mathrm{ext}}}{d\left(V_{\mathrm{ext}}-\phi\right)}=\frac{j_{0} e}{k_{B} T} e^{-\left(z_{\mathrm{O}}-\alpha\right) e \zeta \mathrm{eq} / k_{B} T},
$$

and the Faradaic conductance is redefined as $K=R_{0} / R_{\mathrm{ct}}\left(\zeta^{\mathrm{eq}}\right)$ accordingly. The interaction of the linear response tangential field with the equilibrium Debye layer charge gives rise to a linear response slip velocity

$$
u_{s}^{\prime}=\frac{\epsilon \zeta^{\mathrm{eq}}}{\eta} \partial_{x} \phi^{\prime}
$$

However, because $\phi^{\prime}$ is harmonic in time, this does not give rise to any net pumping. The leading-order time average slip is to second order in $V_{0}$, where the interaction of the induced charge to first order with the tangential field gives rise to a slip velocity of

$$
\left\langle u_{s}^{\prime \prime}\right\rangle=\frac{\epsilon}{\eta}\left\langle\zeta^{\prime} \partial_{x} \phi^{\prime}\right\rangle=-\frac{\epsilon}{4 \eta} \frac{d \zeta}{d\left(V_{\mathrm{ext}}-\phi\right)} \partial_{x}\left|\hat{V}_{\mathrm{ext}}-\hat{\phi}^{\prime}\right|^{2} .
$$

This is fully equivalent to Eq. (28), and the net pumping produced can be obtained directly from the analysis presented earlier in the text. Only now the characteristic fluid velocity becomes

$$
u_{0}=\frac{\epsilon V_{0}^{2}}{\eta \ell_{0}} \frac{d \zeta}{d\left(V_{\mathrm{ext}}-\phi\right)},
$$

where, for a symmetric $z: z$ electrolyte,

$$
\frac{d \zeta}{d\left(V_{\mathrm{ext}}-\phi\right)}=\frac{1}{1+\delta \cosh \left(z e \zeta^{\mathrm{eq}} / 2 k_{B} T\right)} \text {. }
$$

Notice that $u_{0}\left(\zeta^{\text {eq }}\right)$ falls off exponentially with $\zeta^{\text {eq }}$ because $\zeta^{\prime}$ becomes negligible.

The linear response dominates over the higher-order components only at low applied voltage. Specifically, we must require that both $V_{0} \ll C_{d} /\left[d C_{d} / d\left(V_{\text {ext }}-\phi\right)\right]$ and $V_{0}$ $\ll R_{\mathrm{ct}} /\left[d R_{\mathrm{ct}} / d\left(V_{\mathrm{ext}}-\phi\right)\right]$, and a sufficient condition for this is $V_{0} \ll(1+\delta) k_{B} T / z e$. However, because the time average slip velocity vanishes to first order in $V_{0}$, one cannot neglect the second-order tangential field $-\partial_{x} \phi^{\prime \prime}$, which gives rise to an additional component $\left\langle\bar{u}_{s}^{\prime \prime}\right\rangle$ to the slip velocity given by

$$
\left\langle\bar{u}_{s}^{\prime \prime}\right\rangle=\frac{\epsilon \zeta^{\mathrm{eq}}}{\eta} \partial_{x}\left\langle\phi^{\prime \prime}\right\rangle
$$

In the absence of Faradaic currents, the time average potential $\langle\phi\rangle$ vanishes to second and all higher orders within our simple electrokinetic model, as is easily seen by taking the time average of Eq. (9). Otherwise, $\left\langle\phi^{\prime \prime}\right\rangle$ is determined as a solution to the Laplace equation with the boundary condition

$$
0=\sigma \mathbf{n} \cdot \boldsymbol{\nabla}\left\langle\phi^{\prime \prime}\right\rangle-\frac{1}{R_{\mathrm{ct}}}\left\langle\phi^{\prime \prime}\right\rangle+\frac{1}{4} \frac{d^{2} j_{\mathrm{ext}}}{d\left(V_{\mathrm{ext}}-\phi\right)^{2}}\left|\hat{V}_{\mathrm{ext}}-\hat{\phi}^{\prime}\right|^{2}
$$

at the electrodes, where 


$$
\frac{d^{2} j_{\mathrm{ext}}}{d\left(V_{\mathrm{ext}}-\phi\right)^{2}}=-\frac{e}{k_{B} T} \frac{2}{R_{\mathrm{ct}}}\left[\left(z_{\mathrm{O}}-\alpha\right) \frac{d \zeta}{d\left(V_{\mathrm{ext}}-\phi\right)}+\alpha-\frac{1}{2}\right] .
$$

This demonstrates that when Faradaic electrode reactions occur, the actual computation of the time average fluid flow and net pumping for finite intrinsic zeta potential (on the electrodes and/or the insulating channel walls) requires a separate and more elaborate study, which we have to leave open despite its experimental relevance.

\section{NONLINEAR ANALYSIS}

While theoretically it is convenient to work in the lowvoltage limit where the system can be characterized by relatively few dimensionless parameters, most experimental work has been done with driving voltages of a few volts in order to obtain an appreciable fluid motion. However, even with the simple nonlinear model we consider here, the parameter space is large, and we therefore focus on the case of a monovalent symmetric (1:1) electrolyte with no intrinsic zeta potential formed on the electrodes-i.e., $\zeta^{\mathrm{eq}}=0$. Moreover, for the Faradaic electrode reaction we take $z_{\mathrm{O}}=z_{\mathrm{R}}+1$ $=1$ and assume a symmetric transfer coefficient $\alpha=1 / 2$.

We first investigate the system behavior without the presence of the electrode reaction so that the only nonlinearity in our model arises from the nonlinear capacitance of the Debye layer. Then we turn on the electrode reaction and study the full nonlinear model.

\section{A. Dimensionless form for nonlinear analysis}

Beyond the Debye-Hückel limit, the smallest characteristic voltage of the electrokinetic system is the thermal voltage $k_{B} T / e \sim 25 \mathrm{mV}$ and not the driving voltage $V_{0}$. We therefore introduce a new rescaling for the electrical part of the problem:

$$
\phi=\frac{k_{B} T}{e} \tilde{\phi}, \quad V_{0}=\frac{k_{B} T}{e} \tilde{V}_{0}, \quad q=C_{0} \frac{k_{B} T}{e} \tilde{q} .
$$

Apart from this, the scaling remains as in Eq. (21); in particular, we retain $u_{0}=\epsilon V_{0}^{2} / \eta \ell_{0}(1+\delta)$ for the characteristic fluid velocity. Then Eqs. (5), (6), (9), and (15), governing the charging of the Debye layer on the electrodes, reduce to

$$
\begin{gathered}
\tilde{V}_{\mathrm{ext}}-\tilde{\phi}=\tilde{\zeta}-\tilde{q} \delta /(1+\delta), \\
\partial_{t} \tilde{q}=\mathbf{n} \cdot \widetilde{\nabla} \tilde{\phi}+\tilde{j}_{\mathrm{ext}},
\end{gathered}
$$

where $\widetilde{j}_{\text {ext }}$ is the dimensionless Faradaic current given by

$$
\widetilde{j}_{\text {ext }}=2 K e^{-\tilde{\xi} / 2} \sinh \left[\left(\tilde{V}_{\text {ext }}-\widetilde{\phi}\right) / 2\right],
$$

with $K=R_{0} / R_{\text {ct }}$ as before, and where the zeta potential is directly related to the Debye layer charge by

$$
\widetilde{\zeta}(\widetilde{q})=-2 \sinh ^{-1}[\widetilde{q} / 2(1+\delta)] .
$$

On the insulating walls we have again

$$
\mathbf{n} \cdot \widetilde{\nabla} \widetilde{\phi}=0
$$

and, in the bulk,

$$
-\widetilde{\nabla}^{2} \widetilde{\phi}=0
$$

The problem, Eqs. (45)-(50), is solved numerically with the finite-element method using FEMLAB. Due to the nonlinearity, it is not possible to solve the problem with a single complex variable for the base frequency component. Instead we employ a relaxation method, where we represent the periodic solution by a set of equispaced points over one period in time, and calculate the time derivative in Eq. (46) using the leapfrog finite-difference scheme; see Appendix B for details.

\section{B. Nonlinear Debye layer capacitance}

In the absence of Faradaic electrode reactions the only nonlinearity in the model arises from the nonlinear surface capacitance in the Debye layer. Figure 11 shows the pumping velocity $\widetilde{U}$ as a function of the driving frequency $\widetilde{\omega}$ and voltage $\widetilde{V}_{0}$ for $\delta=0.1$ and $K=0$. At the base line for $\widetilde{V}_{0} \ll 1$ we recover the results from the linear analysis with $\tilde{U}$ peaking for $\widetilde{\omega} \sim 1$. However, at higher voltage when the doublelayer capacitance is dominated by the Stern layer, the frequency at which $\tilde{U}$ is maximized drops to $\tilde{\omega} \sim \widetilde{\omega}_{\infty}$, where $\widetilde{\omega}_{\infty}=\tau_{0} / \tau_{\infty}=\delta /(1+\delta)$ is the relaxation frequency at high voltage; cf. Table I.

Figure 11 also shows that the (dimensionless) pumping velocity $\tilde{U}$ falls off at high voltage. This is simply due to the fact that the (physical) electro-osmotic slip velocity $u_{s}$ $\propto \zeta \partial_{x} \phi$ no longer scales as $V_{0}^{2}$. When the driving frequency is not too large, Eqs. (45) and (48) allow us to estimate the zeta potential from

$$
\tilde{V}_{0} \sim \tilde{\zeta}+2 \sinh (\tilde{\zeta} / 2) \delta .
$$

At low voltage this reduces to $\widetilde{\zeta} \sim V_{0} /(1+\delta)$ whereas at high voltage $\tilde{\zeta} \sim 2 \log \left(\tilde{V}_{0} / \delta\right)$. The transition between the two regimes occurs for $\tilde{V}_{0} \sim \delta$ when $\delta \gg 1$ and for $\widetilde{V}_{0} \sim-2 \log \delta$ when $\delta \ll 1$. Hence, for $\widetilde{V}_{0} \gg \delta-2 \log \delta$ we expect $u_{s}$ to scale as $V_{0} \log V_{0}$ rather than $V_{0}^{2}$. This is confirmed by Fig. 12, which shows $\tilde{U}_{\max }$ as a function of $\tilde{V}_{0}$; the data are scaled with $\widetilde{V}_{0}^{2}$ to recover the voltage dependence of the physical pumping velocity:

$$
U=\frac{\epsilon}{\eta \ell_{0}(1+\delta)}\left(\frac{k_{B} T}{e}\right)^{2} \tilde{U} \tilde{V}_{0}^{2} .
$$

We must emphasize at this point that the upper voltage limit $\widetilde{V}_{0}=10^{3}$ in Figs. 11 and 12, corresponding to $V_{0}=25 \mathrm{~V}$, is far outside the range of validity of our simple electrokinetic model: In Sec. VI we discuss a number of strongly nonlinear phenomena that become significant for $V_{0} \gtrsim 125 \mathrm{mV}$ in a typical experiment. Beyond this voltage our results can therefore only provide some characteristic features of the system and should not be regarded as an accurate description. 


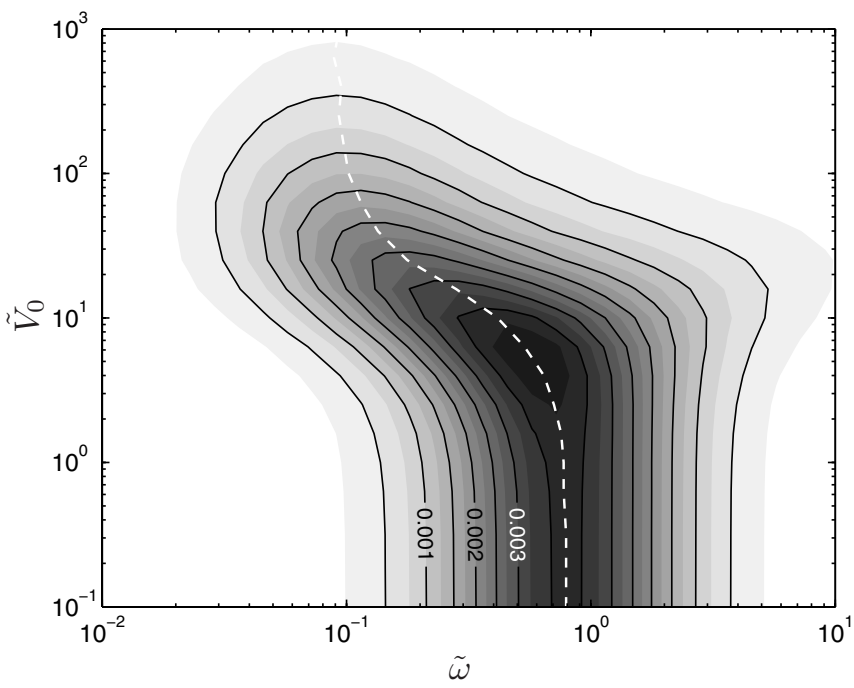

FIG. 11. Contour plot of the pumping velocity $\widetilde{U}$ as a function of frequency $\widetilde{\omega}$ and driving voltage $\widetilde{V}_{0}$ with capacitance ratio $\delta$ $=0.1$ and no Faradaic current, $K=0$. The white dashed line shows the frequency $\widetilde{\omega}_{\max }$ at which $\widetilde{U}$ is maximized as a function of $\widetilde{V}_{0}$. At large driving voltage $\widetilde{\omega}_{\max }$ shifts from $\sim 1$ to $\sim \widetilde{\omega}_{\infty}$, reflecting the larger relaxation time when the double-layer capacitance is dominated by the Stern layer.

\section{Nonlinear Faradaic current injection}

While the nonlinearity induced by the Debye layer capacitance manifests itself relatively slowly as the voltage is increased, we expect a more dramatic effect from the Faradaic current due to the exponential voltage dependence in Eq. (47). Figure 13 shows the pumping velocity $\widetilde{U}$ as a function of driving frequency $\widetilde{\omega}$ and voltage $\tilde{V}_{0}$ for $\delta=0.1$ and $K$ $=0.1$. At the base line for $\widetilde{V}_{0} \ll 1$ we recover the results from the linear analysis in Fig. 8 for $K=0.1$-i.e., no pumping in the low-frequency limit and maximal pumping for $\tilde{\omega} \sim 1$,

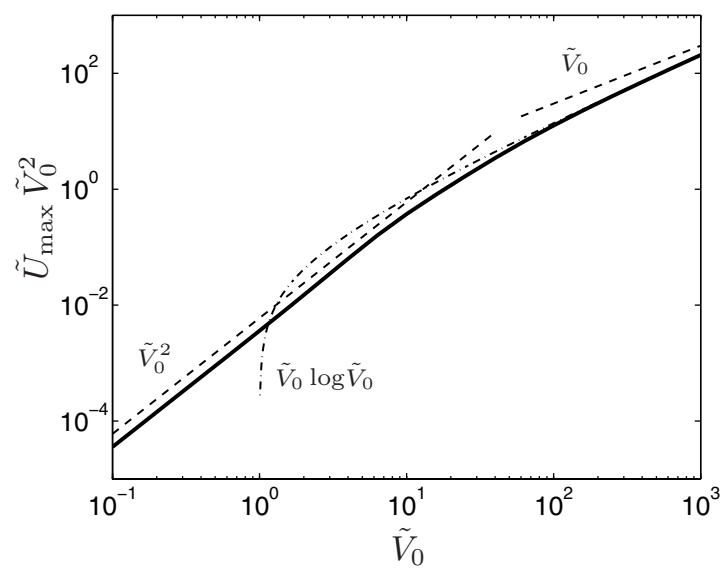

FIG. 12. Maximal pumping velocity $\widetilde{U}_{\max }$ as a function of $\widetilde{V}_{0}$, as evaluated along the white dashed line in Fig. 11. The data are scaled with $\widetilde{V}_{0}^{2}$ to recover the physical dependence on driving voltage. For comparison, the dashed lines show linear and quadratic scaling with $\tilde{V}_{0}$, while the dash-dotted line is a fit to $\tilde{V}_{0} \log \tilde{V}_{0}$.

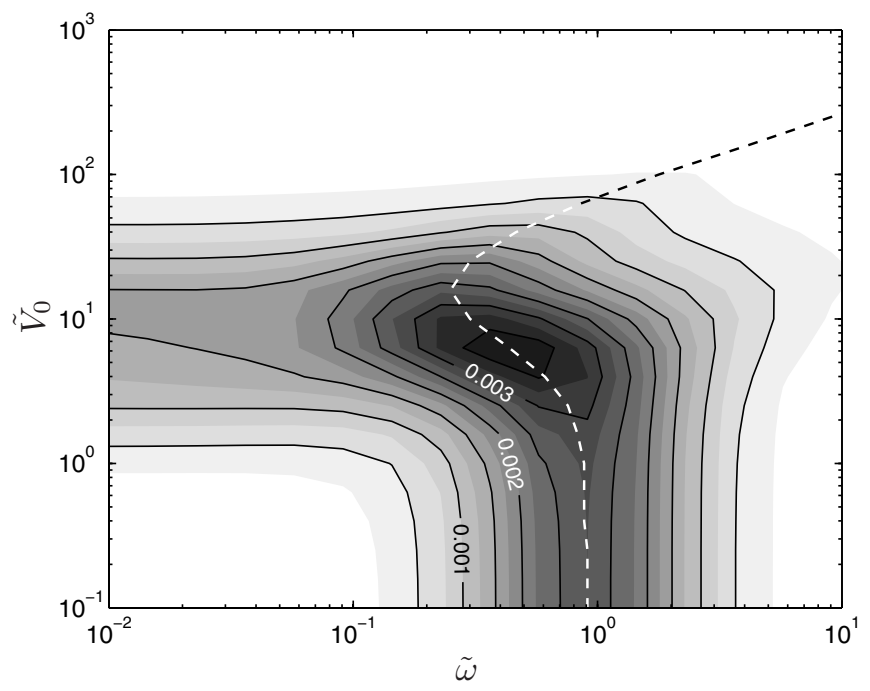

FIG. 13. Contour plot of the pumping velocity $\widetilde{U}$ as a function of driving frequency $\widetilde{\omega}$ and voltage $\widetilde{V}_{0}$ with capacitance ratio $\delta$ $=0.1$ and Faradaic conductance $K=0.1$. The dashed line shows the optimum driving frequency $\widetilde{\omega}_{\max }$ as a function of $\widetilde{V}_{0}$. Notice the pumping in the low-frequency limit for $\tilde{V}_{0} \gtrsim 1$.

with a peak value of $\tilde{U}$ less than 0.003 . However, for $\widetilde{V}_{0}$ $\gtrsim 1$ we do observe pumping at low frequency, whereas $\tilde{U}$ falls off rapidly for all $\tilde{\omega}$ when $\tilde{V}_{0} \gg 10$. The figure also shows that at low voltage the optimal driving frequency $\widetilde{\omega}_{\max }$ follows the same pattern as in Fig. 11 , but for $\tilde{V}_{0} \gtrsim 10$ it bends sharply up towards higher frequency.

Qualitatively it is not so difficult to see how the pumping at low frequency arises: At low voltage the (magnitude of the) charge density in the Debye layer on the narrow electrode is a factor of $W_{2} / W_{1}$ larger than on the wide electrode to ensure overall charge conservation. Correspondingly, also the double-layer potential drop $\widetilde{V}_{\text {ext }}-\widetilde{\phi}$ is larger on the narrow electrode, such that the exponential increase in the Faradaic current [cf. Eq. (47)] starts off earlier there. Effectively, this makes the Faradaic conductance larger on the narrow electrode than on the wide one, and according to Fig. 9 from our linear analysis this should indeed give rise to pumping in the forward direction at low frequency.

Figure 14 shows in more detail the voltage dependence of the pumping velocity at low frequency $\tilde{\omega} \ll 1$. For $\tilde{V}_{0} \lesssim 1$ the net pumping grows rapidly with $\tilde{V}_{0}$. Apart from the intrinsic $\tilde{V}_{0}^{2}$ scaling, the time average asymmetry in effective Faradaic conductance between the two electrodes grows roughly as $\widetilde{V}_{0}^{2}$ as well, yielding an overall $\widetilde{V}_{0}^{4}$ voltage dependence. At larger voltage we find that the pumping levels off to become almost independent of $\widetilde{V}_{0}$. This behavior can be partially understood by considering the scaling of $\widetilde{\zeta}$ and the tangential field in this limit: When the frequency is low enough that a steady state is established with the Ohmic and Faradaic currents balancing, we can write the applied voltage $\widetilde{V}_{0}$ as the sum of the potential drop across the double layer $\widetilde{V}_{\text {ext }}-\widetilde{\phi}$ and the poten- 


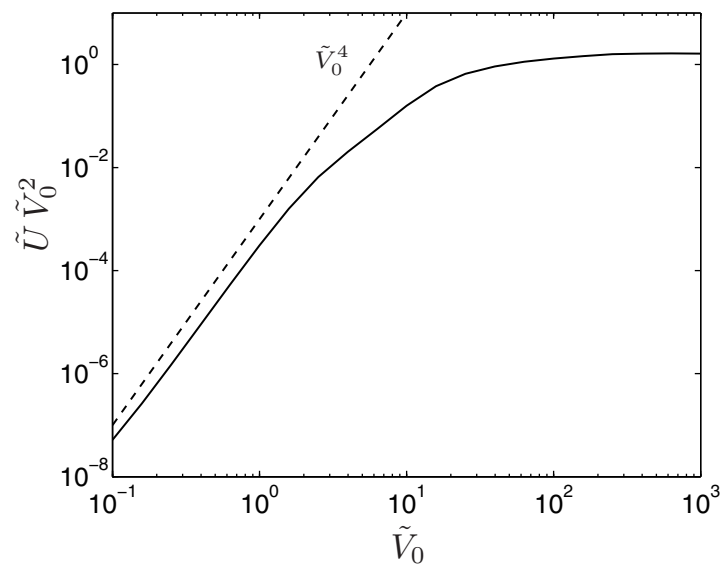

FIG. 14. Pumping velocity $\widetilde{U}$ as a function of $\widetilde{V}_{0}$ in the lowfrequency limit $\widetilde{\omega} \ll 1$. The data are scaled with $\widetilde{V}_{0}^{2}$ to recover the physical dependence on driving voltage.

tial drop across the bulk electrolyte when an Ohmic current equal to $\tilde{j}_{\text {ext }}$ is passed through it-i.e.,

$$
\begin{aligned}
\tilde{V}_{0} \sim & \left(\widetilde{V}_{\mathrm{ext}}-\widetilde{\phi}\right)+\tilde{j}_{\mathrm{ext}} \sim\left(\widetilde{V}_{\mathrm{ext}}-\tilde{\phi}\right)+2 K e^{-\tilde{\xi} / 2} \\
& \times \sinh \left[\left(\widetilde{V}_{\mathrm{ext}}-\widetilde{\phi}\right) / 2\right] .
\end{aligned}
$$

If we neglect for simplicity the $\widetilde{\zeta}$-dependent exponential prefactor, we find immediately that $\widetilde{V}_{\text {ext }}-\widetilde{\phi} \sim \widetilde{V}_{0} /(1+K)$ at low voltage $\tilde{V}_{0} \ll K-2 \log K$, changing to $\tilde{V}_{\text {ext }}-\widetilde{\phi}$ $\sim 2 \log \left(\widetilde{V}_{0} / K\right)$ at higher voltage. In effect, both the tangential field $\partial_{\tilde{x}} \widetilde{\phi}=-\partial_{\tilde{x}}\left(\widetilde{V}_{\text {ext }}-\widetilde{\phi}\right)$ and the zeta potential $\widetilde{\zeta} \approx\left(\widetilde{V}_{\text {ext }}\right.$ $-\phi) /(1+\delta)$ go as $\log \widetilde{V}_{0}$ at large voltage, and we would expect the (physical) electro-osmotic slip velocity $u_{s} \propto \zeta \partial_{x} \phi$ to grow as slowly as $\left(\log V_{0}\right)^{2}$ with driving voltage.

Finally, we note that since the electrode reaction is very fast at large voltage, the "low-frequency" limit where the Faradaic and Ohmic currents balance extends higher and higher in frequency as the driving voltage is increased. Therefore, the characteristic behavior from Fig. 14 with the pumping velocity leveling off dominates even for $\widetilde{\omega} \sim 1$ when $\widetilde{V}_{0} \gtrsim 10^{2}$. And when the optimal driving frequency in Fig. 13 shifts towards higher frequency for $\widetilde{V}_{0} \gtrsim 10$, it is essentially a trace of the point at which the Faradaic current starts to dominate.

\section{DISCUSSION}

Our numerical study of the simple electrokinetic model has brought about a number of interesting theoretical predictions that we briefly summarize below and compare to experimental observations reported in the literature. In particular, we comment on the extent to which our results agree or disagree with the experiments and discuss a number of (nonlinear) effects not included in our model that could account for some of the more exotic experimental observations.

\section{A. Confinement}

Our analysis has shown that vertical confinement of the pumping channel does not mask the asymmetry of the device and destroy the ability to pump, as one might have worried, but instead enhances the pumping velocity by roughly a factor of 2 and causes the optimal driving frequency to shift down inversely proportional to $L / H$. In all experimental studies to date, the confinement has been relatively weak with $L / H \leqq 2$, whereas our results indicate that confinement becomes significant only for $L / H>2$. It is important to keep in mind, though, that while the pumping velocity $U$ does not depend much on confinement, the maximal flow rate $Q_{\max }$ $=H U / 2$ that the pump can deliver and the maximal backpressure $\Delta p_{\max } \propto U / H^{2}$ it can sustain certainly do. The optimal choice of channel height therefore depends strongly on whether the pump is intended to work as a pressure or current generator [27].

\section{B. Nonlinear double-layer capacitance}

In the absence of Faradaic electrode reactions, where the sole nonlinearity in our model is due to the nonlinear capacitance of the Debye layer, we find that as the driving voltage $V_{0}$ is increased well beyond the thermal voltage $k_{B} T / e$, the relaxation frequency for the electrokinetic system, and hence also the optimal frequency for pumping, drops from $\omega_{0}$ $=1 / R_{0} C_{0}$ to $\omega_{\infty}=1 / R_{0} C_{s}=\omega_{0} \delta /(1+\delta)$, where $\delta=\epsilon / \lambda_{D} C_{s}$ is the surface capacitance ratio. At the same time, the scaling of the pumping velocity with driving voltage changes from $V_{0}^{2}$ to $V_{0} \log V_{0}$ because the zeta potential entering the Helmholtz-Smoluchowski slip velocity becomes only a small fraction of the overall potential drop across the double layer.

Experimentally, Brown et al. reported that the optimal driving frequency $f_{\max }$ dropped from $3 \mathrm{kHz}$ at $0.2 \mathrm{~V}_{\mathrm{rms}}$ to $1 \mathrm{kHz}$ at $1.2 \mathrm{~V}_{\mathrm{rms}}$ [6]. For their particular electrolyte and electrode geometry the relaxation frequency at low voltage was expected to be $f_{0}=\omega_{0} / 2 \pi \simeq(1+\delta)$ $\times 2 \mathrm{kHz}$. The observed downshift in optimal frequency therefore fits well with our model predictions if we assume a capacitance ratio of $\delta \simeq 0.5$. Likewise, Studer $e t$ al. found the optimal driving frequency around $1 \mathrm{kHz}$ for driving voltages larger than $1 \mathrm{~V}_{\text {rms }}$ with similar electrolyte and electrode geometry [11]. The electrode material in those experimental studies was uncoated gold and platinum, respectively, consistent with a large Stern layer capacitance and hence small $\delta$.

Green et al. mapped out the entire fluid velocity field over a single pair of gold electrodes with titanium coating [4]. The titanium spontaneously forms a thin oxide layer which was intended to inhibit Faradaic electrode reactions in the experiment. The observed velocity magnitude and frequency dependence at $500 \mathrm{mV}$ driving voltage was matched with linear theory predictions by assuming a capacitance ratio of $\delta$ $=3$. It is remarkable that this value was obtained for both a $0.15 \mathrm{mM}$ and a $0.6 \mathrm{mM} \mathrm{KCl}$ solution: Naively, assuming a constant value for the Stern layer capacitance $C_{s}$ one would expect $\delta=\epsilon / \lambda_{D} C_{S}$ to depend on the electrolyte concentration through $\lambda_{D}$ [28].

Cahill et al. observed ac electroosmotic fluid motion due to traveling-wave electric fields induced on a four-phase in- 
terdigitated electrode array and found an accurate $V_{0}^{2}$ scaling for $100-500 \mathrm{mV}$ driving voltage, although the pumping velocity peak position and magnitude were smaller than the linear theory predictions [12]. In their experiments the electrodes were coated with a 50-nm-thick Teflon-like insulating layer, yielding a capacitance ratio of $\delta=75-150$ depending on the electrolyte concentration. Hence, they remained in the linear regime over the entire voltage range applied. Ramos et al. performed a similar study using titanium-coated gold electrodes [13]. Their optimal driving frequency around $2 \mathrm{kHz}$ was matched with the linear theory by assuming $\delta$ $=1.5$, while the maximum pumping velocity at low frequency was an order of magnitude below the prediction from linear theory.

Generally, the experimentally observed pumping velocities tend to be smaller than the linear theory prediction and sometimes resemble more an affine dependence than a quadratic scaling with $V_{0}[10,11]$. This is at least in qualitative agreement with our nonlinear model results because it is difficult to distinguish $V_{0}$ from $V_{0} \log V_{0}$ over the limited voltage range used experimentally.

\section{Faradaic current injection}

Our linear analysis at low driving voltage shows that when Faradaic electrode reactions occur, the pumping is strongly suppressed for $R_{\mathrm{ct}} \ll R_{0}$ due to "short-circuiting" of the double layer, where $R_{\mathrm{ct}}$ is the charge-transfer resistance characterizing the electrode reaction and $R_{0}$ is the bulk electrolyte Ohmic resistance. When $R_{\mathrm{ct}} \sim R_{0}$ the Faradaic current injection merely imbalances the otherwise perfect screening at low frequency and therefore induces electro-osmotic fluid motion. Moreover, we predict that the direction of the pumping in the low-frequency limit depends on the relative magnitude of $R_{\mathrm{ct}}$ on the narrow and wide electrodes, respectively: If $R_{\mathrm{ct}}$ is smaller on the narrow electrodes than on the wide ones, the pumping at low frequency is in the forward direction, whereas it is in the reverse direction otherwise. In the symmetric special case when $R_{\mathrm{ct}}$ is equal on both electrodes there is no net pumping at low frequency.

When the driving voltage is increased, the nonlinearity in the Faradaic current injection induces an effective asymmetry in the charge-transfer resistance and we obtain pumping in the forward direction at low frequency, even if the intrinsic surface properties are symmetric. Moreover, at even larger voltage our nonlinear analysis shows that the pumping velocity levels off to a constant value. Using the typical parameter values from Table I and $R_{0} / R_{\mathrm{ct}}=0.1$ as in Fig. 14 , the magnitude of the pumping velocity at this plateau is roughly $U \sim 100 \mu \mathrm{m} / \mathrm{s}$.

The observation of electrode degradation and bubble formation from electrolysis is experimental evidence that Faradaic reactions do occur above $1.5-4.0 \mathrm{~V}_{\text {rms }}$, depending on the driving frequency and electrode material [8-11,14]. Moreover, Studer et al. observed that the pumping direction was reversed when the driving frequency was increased above $20 \mathrm{kHz}$ and the voltage above $3 \mathrm{~V}_{\text {rms }}$ [11]. Ramos et $a l$. also found reversal of the pumping direction on an asymmetric electrode array, as well as a similar reversal on a traveling-wave array for driving voltages above $2 \mathrm{~V}$ and frequencies around $1 \mathrm{kHz}[13,14]$. And Lastochkin et al. reported that the direction of the time average slip velocity on an array of T-shaped electrodes was reversed as compared to the direction usually expected for ac electroosmosis [16].

We note that the reversal of the pumping direction that we predict at low frequency is essentially due to an imbalance between net pumping contributions of opposite signs from the two electrodes. We never find reversal of the time average slip direction within our simple electrokinetic model. It is possible that the pumping reversal observed by Studer et al. could be due to imbalance between the net pumping contributions from the two electrodes: Although our nonlinear analysis predicts pumping in the forward direction, there could be other nonlinear effects leading to imbalance into the reverse direction. However, Ramos et al. observed pumping reversal on a traveling-wave array where all the electrodes are identical-this rules out any imbalance between the contributions from individual electrodes in their setup. And the reversal of the time average slip direction observed by Lastochkin et al. is certainly a different phenomenon.

Lastochkin et al. argued that strong Faradaic current injection in their system would reverse the polarity of the charge in the Debye layer and hence the direction of the slip. Based on the simple circuit model for the electrokinetic system from Fig. 2, it is difficult to see how the potential drop across (and hence the charge in) the double layer could change sign, regardless of how small $R_{\mathrm{ct}}$ becomes. However, since the driving frequency in their experiments was in the $\mathrm{MHz}$ range, which is of the order of the Debye frequency for the electrolyte, the Debye layer cannot be assumed to be in local equilibrium and Eqs. (4) and (16) are no longer valid.

Finally, we recall that for simplicity we have been focusing here on the case with no intrinsic zeta potential $\zeta^{\text {eq }}$ on the electrodes and/or the insulating channel walls. Our analysis in Sec. IV F indicates that when Faradaic electrode reactions are present, the behavior for nonzero $\zeta^{\text {eq }}$ needs to be studied more carefully, especially in the nonlinear regime.

\section{Effect of mass transfer on reaction kinetics}

One important effect that we have neglected in our model is the influence of mass transfer on the electrode reaction kinetics. When the oxidized and reduced species $\mathrm{O}$ and $\mathrm{R}$ are consumed or produced at the electrode by the Faradaic reaction, their local concentrations $c_{\mathrm{O}}$ and $c_{\mathrm{R}}$ become different from the bulk concentrations $c_{\mathrm{O}}^{*}$ and $c_{\mathrm{R}}^{*}$, respectively. Now, the lower $c_{\mathrm{O}}$ drops at the electrode, the more difficult it becomes to run the reaction forward, and vice versa for $c_{R}$. This can be modeled as an additional impedance $Z(\omega)$, called the Warburg impedance, in series with the charge-transfer resistance $R_{\mathrm{ct}}$ in Fig. 2 [18]. The transport of $\mathrm{O}$ and $\mathrm{R}$ from the bulk to the surface is by diffusion, and the characteristic distance that the $n$th species can diffuse over one cycle of the driving voltage is $\ell_{n}(\omega)=\sqrt{D_{n} / \omega}$, where $D_{n}$ is the diffusion constant. If this distance is small compared to the characteristic dimension of the electrodes $\ell_{0}$, the diffusion process is essentially one dimensional and the (area specific) Warburg impedance takes the form 


$$
Z(\omega)=\frac{k_{B} T}{\sqrt{2} e^{2}}\left[\frac{1}{c_{\mathrm{O}}^{*} \sqrt{D_{\mathrm{O}}}}+\frac{1}{c_{\mathrm{R}}^{*} \sqrt{D_{\mathrm{R}}}}\right] \frac{1-i}{\sqrt{\omega}} .
$$

Notice that $Z(\omega)$ increases at low frequency because the reactants need to diffuse longer. However, at very low frequency when $\ell(\omega) \gg \ell_{0}$-i.e., $\omega \ll D / \ell_{0}^{2}$ - the diffusion layer extends much longer than the separation between the electrodes and the Warburg impedance levels off to a constant

$$
Z_{0} \sim \frac{k_{B} T}{e^{2}}\left[\frac{\ell_{0}}{c_{\mathrm{O}}^{*} D_{\mathrm{O}}}+\frac{\ell_{0}}{c_{\mathrm{R}}^{*} D_{\mathrm{R}}}\right] .
$$

We note that $Z_{0} \gtrsim R_{0}=\ell_{0} / \sigma$, where the electrolyte conductivity is given by $\sigma=\Sigma_{n} D_{i} c_{n}^{*} z_{n}^{2} e^{2} / k_{B} T$. Therefore, even if $R_{\mathrm{ct}}$ $\ll R_{0}$, it is not possible to short-circuit the double layer for $\omega \ll D / \ell_{0}^{2}$, because it is the larger of $R_{\mathrm{ct}}$ and $Z_{0}$ that dominates. More generally, the effect of mass transfer is to make the short-circuiting of the double layer discussed in Secs. IV E and V C less dramatic. Still, we do expect the prediction of the pumping direction based on Fig. 9 to hold for $\widetilde{\omega}$ $\ll \omega_{0}$ and $R_{\text {ct }} \gtrsim R_{0}$.

As the driving voltage is increased, the Faradaic current can become so strong that the concentrations of the reactants at the electrodes differ significantly from their equilibrium values. Ultimately, as one of them approaches zero, the reaction stagnates. The current at which this occurs is termed the limiting current [18], and it is given by the maximal rate at which the reactants can be transported from the bulk to the electrodes by diffusion. Hence, for the forward reaction the limiting current is $j_{\mathrm{O}}^{\mathrm{lim}} \sim e D_{\mathrm{O}} c_{\mathrm{O}}^{*} / \ell_{\mathrm{O}}(\omega)$. Assuming the frequency is low enough that the Ohmic and Faradaic currents are balancing-i.e., $j_{\text {ext }} \sim \sigma V_{0} / \ell_{0}$-we find that the limiting current is reached around $V_{0} \sim e D_{\mathrm{O}} c_{\mathrm{O}}^{*} \ell_{0} / \sigma \ell_{\mathrm{O}}(\omega)$. For the typical parameter values from Table I and $\omega \sim \omega_{0}, D_{\mathrm{O}}$ $\sim 10^{-9} \mathrm{~m}^{2} / \mathrm{s}$, and $c_{\mathrm{O}}^{*} \sim 0.1 \mathrm{mM}$, this corresponds to $V_{0}$ $\sim 100 \mathrm{mV}$. Beyond this voltage, the concentration $c_{\mathrm{O}}$ tends to zero at the electrode and the Faradaic current levels off at the limiting value. If there is no supporting electrolyte, this makes the conductivity in the diffusion layer differ significantly from the bulk value and the Debye layer is driven out of local equilibrium and expands in width to $\sim \ell_{0}^{1 / 3} \lambda_{D}^{2 / 3}$ $[30,31]$. However, if there is an excess of supporting electrolyte that does not participate in the electrode reaction, the diffusion layer should remain charge neutral and the conductivity largely unaffected. In this case the system will be dominated by capacitive charging and behave as if no Faradaic reaction is taking place. This underlines the potential role of the specifics of the electrolyte and electrode material, known to play a decisive role in a few electrically generated phenomena [32,33].

\section{E. Surface conduction in the Debye layer}

At large voltage the Debye layer can accumulate enough charge that the mean ion density, and hence the conductivity, becomes significantly larger than in the bulk electrolyte. In this case one cannot neglect lateral surface currents in Eq. (9). This is quantified by the Dukhin number $\mathrm{Du}=\sigma_{s} / \sigma \ell_{0}$, where $\sigma_{s}$ is the surface conductivity in the Debye layer, incorporating both migration and electro-osmotic convection of charge, $\sigma$ is the bulk conductivity, and $\ell_{0}$ is the characteristic geometrical length scale. For a symmetric monovalent (1:1) electrolyte the Dukhin number can be expressed as [17]

$$
\mathrm{Du}=\left[1+\left(\frac{k_{B} T}{e}\right)^{2} \frac{2 \epsilon}{\eta D}\right] \frac{\lambda_{D}}{\ell_{0}} 4 \sinh ^{2}\left(\frac{e \zeta}{4 k_{B} T}\right) .
$$

Taking $\zeta \sim V_{0} /(1+\delta)$ and using typical parameter values from Table I, we find that surface conductance becomes significant and $\mathrm{Du} \gtrsim 1$ for $V_{0} \gtrsim 250 \mathrm{mV}$. Generally, we expect surface currents to smear out the charge distribution across the electrodes, reducing the tangential field and hence the electroosmotic slip and pumping velocity. We do not anticipate that this alone could be the mechanism responsible for reversal of the time average slip velocity or the direction of net pumping.

\section{F. Local salt depletion at electrodes}

Another issue relating to the massive accumulation of ions in the Debye layer at large voltage is discussed by $\mathrm{Ba}-$ zant et al. [19]—namely, that of where those ions come from. For a symmetric monovalent (1:1) electrolyte we define the excess amount of neutral salt absorbed in the Debye layer as

$$
\begin{aligned}
w(x, t) & =\frac{1}{2} \int_{\text {Debye layer }}\left(c_{+}+c_{-}-2 c^{*}\right) d y \\
& =c^{*} \lambda_{D} 4 \sinh ^{2}\left(\frac{e \zeta}{4 k_{B} T}\right)
\end{aligned}
$$

This salt is periodically taken up and released again between the Debye layer and a diffusion zone of width $\ell(\omega)=\sqrt{D / \omega}$. However, when the amount of salt absorbed in the Debye layer approaches the total amount $c^{*} \ell(\omega)$ available in the diffusion zone, we get local depletion of salt and our assumption of uniform electrolyte concentration throughout the bulk breaks down. This occurs for

$$
\zeta \sim \frac{k_{B} T}{e} 2 \log \left(\ell(\omega) / \lambda_{D}\right)=\frac{k_{B} T}{e} \log \left(\omega_{D} / \omega\right),
$$

where we wrote $\omega_{D}=D / \lambda_{D}^{2}$. Taking again $\zeta \sim V_{0} /(1+\delta)$ and using typical parameter values from Table I, we find that salt depletion around the relaxation frequency $\omega_{0}$ becomes an issue for $V_{0} \gtrsim 125 \mathrm{mV}$.

There are several interesting phenomena arising when the approximation of uniform bulk electrolyte concentration and conductivity breaks down. Among other things, this would change the local Debye length as seen from the electrode surface and give rise to space charge like $\rho \sim \epsilon \partial_{y} \phi \partial_{y} \log (\sigma)$ in the diffusion layer [32]. While the amount of space charge in the diffusion layer would typically be much smaller than in the Debye layer, it interacts with the fluid in a place more remotely from the wall and is therefore more efficient in setting the fluid in motion globally. We are currently studying in more detail the role of this nonlinear mechanism for inducing fluid motion. 


\section{CONCLUSION}

We have extended existing theory for ac electrokinetic pumping to account for vertical confinement of the pumping channel, nonlinear surface capacitance of the double layer, and current injection from Faradaic electrode reactions in both a linear and a nonlinear scheme. For our particular model system of an asymmetric electrode array micropump we have obtained a number of results that we subsequently have interpreted using simple physical arguments. As these arguments are more general than the specific model, we expect our results to hold with some generality for other similar electrokinetic systems. We therefore believe that our work will be useful for design of electrokinetic micropumps.

Our results compare well with experiments in many aspects but there still remains unexplained phenomena-e.g., the reversal of the net pumping or the time average slip direction. This points out the need for further studies of (other) nonlinear phenomena to fully understand the complex electrohydrodynamic system.

\section{APPENDIX A}

We give here a proof that there can be no net pumping at low frequency when the electrode surface properties are symmetric and we stay within the Debye-Hückel limit. Consider a device where the capacitance ratio $\delta(\tilde{x})$ and inverse charge-transfer resistance $K(\tilde{x})$ vary across the electrodes. The electro-osmotic slip velocity becomes

$$
\tilde{u}_{s}=\frac{1+\delta_{0}}{1+\delta(\tilde{x})}\left(\tilde{V}_{\mathrm{ext}}-\tilde{\phi}\right) \partial_{\tilde{x}} \tilde{\phi}
$$

where $\delta_{0}$ is the capacitance ratio used in the rescaling of the problem, and Eq. (25) describing the charge balance in the Debye layer generalizes to

$$
\mathbf{n} \cdot \widetilde{\nabla} \hat{\phi}=\left(i \widetilde{\omega} \frac{1+\delta_{0}}{1+\delta(\widetilde{x})}+K(\widetilde{x})\right)\left(\hat{\phi}-\hat{V}_{\mathrm{ext}}\right) .
$$

Combining these we can write the time average slip as

$$
\begin{aligned}
\left\langle\tilde{u}_{s}\right\rangle & =\frac{1+\delta_{0}}{1+\delta(\widetilde{x})} \frac{1}{2} \operatorname{Re}\left[\left(\hat{V}_{\mathrm{ext}}-\hat{\phi}\right) \partial_{\tilde{x}} \hat{\phi}^{*}\right] \\
& =-\frac{1}{2} \operatorname{Re}\left[\frac{\mathbf{n} \cdot \widetilde{\nabla} \hat{\phi}}{i \widetilde{\omega}+\kappa(\tilde{x})} \partial_{\tilde{x}} \hat{\phi}^{*}\right],
\end{aligned}
$$

where $\kappa(\tilde{x})=K(\widetilde{x})[1+\delta(\widetilde{x})] /\left(1+\delta_{0}\right)$. In the high-symmetry case where $\kappa(\tilde{x})$ is a constant independent of $\tilde{x}$, the net pumping velocity $\tilde{U}$ can be manipulated as follows:

$$
\tilde{U}=\frac{1}{\tilde{L}} \int_{\text {electrodes }}\left\langle\tilde{u}_{s}\right\rangle d \tilde{x}=\frac{1}{2 \tilde{L}} \int_{\partial \mathcal{V}}-\operatorname{Re}\left[\frac{\mathbf{n} \cdot \widetilde{\nabla} \hat{\phi}}{i \tilde{\omega}+\kappa} \partial_{\tilde{x}} \hat{\phi}^{*}\right] d \widetilde{s},
$$

where $\partial \mathcal{V}$ denotes the boundary of the computational domain and we used the fact that $\mathbf{n} \cdot \widetilde{\nabla} \hat{\phi}$ vanishes on insulating walls and cancels over periodic boundaries. Then, using Gauss' law and recalling that $\mathbf{n}$ is a unit normal pointing out of the electrodes and into $\mathcal{V}$, we find

$$
\begin{aligned}
& \tilde{U}=\frac{1}{2 \tilde{L}} \operatorname{Re}\left[\frac{1}{i \widetilde{\omega}+\kappa} \int_{\mathcal{V}} \tilde{\nabla} \cdot\left(\widetilde{\nabla} \hat{\phi} \partial_{\tilde{x}} \hat{\phi}^{*}\right) d \widetilde{\mathbf{r}}\right] \\
& =\frac{1}{2 \tilde{L}} \operatorname{Re}\left[\frac{1}{i \widetilde{\omega}+\kappa} \int_{\mathcal{V}} \tilde{\nabla} \hat{\phi} \cdot \widetilde{\nabla}\left(\partial_{\tilde{x}} \hat{\phi}^{*}\right) d \mathbf{r}\right] \\
& =\frac{1}{2 \tilde{L}} \int_{\mathcal{V}} \frac{\frac{1}{2} \kappa \partial_{\tilde{x}}\left(\widetilde{\nabla} \hat{\phi} \cdot \widetilde{\nabla} \hat{\phi}^{*}\right)+\widetilde{\omega} \operatorname{Im}\left[\widetilde{\nabla} \hat{\phi} \cdot \widetilde{\nabla}\left(\partial_{\tilde{x}} \hat{\phi}^{*}\right)\right]}{\kappa^{2}+\widetilde{\omega}^{2}} d \widetilde{\mathbf{r}} \\
& =\frac{1}{2 \widetilde{L}} \frac{\tilde{\omega}}{\kappa^{2}+\widetilde{\omega}^{2}} \operatorname{Im}\left[\int_{\mathcal{V}} \widetilde{\nabla} \hat{\phi} \cdot \widetilde{\nabla}\left(\partial_{\tilde{x}} \hat{\phi}^{*}\right) d \widetilde{\mathbf{r}}\right] \text {. }
\end{aligned}
$$

Here Eq. (A6) follows from $\widetilde{\nabla}^{2} \hat{\phi}=0$, Eq. (A7) follows from simple complex arithmetics, and finally, Eq. (A8) holds because the integral of the term $\partial_{\tilde{x}}\left(\widetilde{\nabla} \hat{\phi} \cdot \nabla \hat{\phi}^{*}\right)$ cancels out due to periodicity.

The final result in Eq. (A8) clearly shows that regardless of $\kappa$, the net pumping $\widetilde{U}$ goes to zero in the low-frequency limit $\widetilde{\omega} \rightarrow 0$. The apparent singular behavior for $\kappa=0$ is only apparent because the screening is then complete and $\widetilde{\nabla} \hat{\phi}$ zero in the bulk. Hence, our analysis rules out any net pumping in the low-frequency limit of our simple model when the electrode surface properties are symmetric in the sense of making the parameter $\kappa$ independent of $\tilde{x}$.

\section{APPENDIX B}

The nonlinear problem is converted to weak form by multiplying a test function $\varphi(\widetilde{\mathbf{r}})$ for $\widetilde{\phi}$ on Eq. (50), integrating over the computational domain $\mathcal{V}$, and using Gauss' law with Eqs. (46) and (49) to eliminate $\mathbf{n} \cdot \widetilde{\nabla} \widetilde{\phi}$. Hence, for $\widetilde{\phi}$ to be a weak solution we require that

$$
\int_{\mathcal{V}} \widetilde{\nabla} \varphi \cdot \nabla \widetilde{\phi} d \widetilde{\mathbf{r}}+\int_{\text {electrodes }} \varphi\left[\partial_{\tilde{t}} \tilde{q}-\widetilde{j}_{\text {ext }}(\widetilde{q})\right] d \tilde{x}=0
$$

for all $\varphi$ and at all times. The constraint, Eq. (45), on the potential drop across the double layer is satisfied by multiplying a test function $\varrho(\tilde{x})$ for $\tilde{q}$ and requiring that

$$
\int_{\text {electrodes }} \varrho\left[\tilde{V}_{\text {ext }}-\tilde{\phi}-\tilde{\zeta}(\tilde{q})+\tilde{q} \delta /(1+\delta)\right] d \tilde{x}=0
$$

for all $\varrho$ and at all times. The weak problem is discretized using the Galerkin method; i.e., we expand $\widetilde{\phi}$ on a finite set of basis functions $\left\{\varphi_{n}(\widetilde{\mathbf{r}})\right\}_{n=1}^{N_{\phi}}$ as

$$
\widetilde{\phi}(\widetilde{\mathbf{r}}, \widetilde{t}) \approx \sum_{n=1}^{N_{\phi}} \widetilde{\phi}_{n}(\widetilde{t}) \varphi_{n}(\widetilde{\mathbf{r}})
$$

and similarly for $\tilde{q}$ on $\left\{\varrho_{n}(\tilde{x})\right\}_{n=1}^{N_{q}}$, and we use those same basis sets as test functions in Eqs. (B1) and (B2). Further, the solution is periodic in time, so we discretize on a set of $M$ equispaced points $\widetilde{t}_{m}=m \Delta \widetilde{t}, m=1,2, \ldots, M$, over one period, and use the leapfrog finite-difference scheme $\partial_{t} \widetilde{q}_{n}\left(\widetilde{t}_{m}\right)$ $\approx\left[\widetilde{q}_{n}\left(\widetilde{t}_{m+1}\right)-\widetilde{q}_{n}\left(\widetilde{t}_{m-1}\right)\right] / 2 \Delta \widetilde{t}$ to evaluate the time derivative in Eq. (B1). 
The full coupled problem for all $M$ time steps is solved numerically by damped Newton iterations, using the commercial finite-element software FEMLAB to define the basis functions $\varphi_{n}(\widetilde{\mathbf{r}})$ and $\varrho_{n}(\widetilde{x})$ and to evaluate the Jacobian matrix of the discretized problem. In the frequency-voltage maps in Figs. 11 and 13 there are five data points per decade for both frequency and voltage. The convergence of the Newton iterations is accelerated by a continuation scheme where the converged solution at one frequency is used as initial guess for the next solution at higher frequency and the same voltage; at the lowest frequency, we use the solution from lower voltage as initial guess when stepping to higher voltage.

With nonlinear Debye layer capacitance but no Faradaic currents, we obtain fairly accurate results with a coarse time resolution of $M=16$ : When compared to a fine resolution result at $M=64$, the maximal relative error on $\tilde{U}$ is less than $1 \%(3 \%)$ for $\widetilde{V}_{0} \leqslant 10^{1}\left(10^{3}\right)$, whereas the maximal relative error on $\widetilde{q}$ is about twice as large. Figures 11 and 12 show the results for $M=64$. With Faradaic current injection, the charging and decharging of the Debye layer becomes very rapid at high voltage, which makes the solution more demanding: Comparing results for $M=64$ and $M=128$ we find that the maximal relative error on $\widetilde{U}$ is less than $0.1 \%(1.5 \%)$ for $\widetilde{V}_{0} \leqslant 10^{1}\left(10^{3}\right)$, but that the corresponding error on $\widetilde{q}$ is as large as $0.5 \%(20 \%)$. However, one should keep in mind that at high voltage our weakly nonlinear model also becomes physically inadequate for reasons discussed in Secs. VI D-VI F. Figures 13 and 14 show results for $M=128$.
[1] H. A. Stone, A. D. Stroock, and A. Ajdari, Annu. Rev. Fluid Mech. 36, 381 (2004).

[2] N. G. Green, A. Ramos, A. Gonzalez, H. Morgan, and A. Castellanos, Phys. Rev. E 61, 4011 (2000).

[3] A. Gonzalez, A. Ramos, N. G. Green, A. Castellanos, and H. Morgan, Phys. Rev. E 61, 4019 (2000).

[4] N. G. Green, A. Ramos, A. Gonzalez, H. Morgan, and A. Castellanos, Phys. Rev. E 66, 026305 (2002).

[5] A. Ajdari, Phys. Rev. E 61, R45 (2000).

[6] A. B. D. Brown, C. G. Smith, and A. R. Rennie, Phys. Rev. E 63, 016305 (2000).

[7] S. Debesset, C. J. Hayden, C. Dalton, J. C. T. Eijkel, and A. Manz (unpublished).

[8] V. Studer, A. Pepin, Y. Chen, and A. Ajdari, Microelectron. Eng. 61-62, 915 (2002).

[9] M. Mpholo, C. G. Smith, and A. B. D. Brown, Sens. Actuators B 92, 262 (2003).

[10] S. Debesset, C. J. Hayden, C. Dalton, J. C. T. Eijkel, and A. Manz, Lab Chip 4, 396 (2004).

[11] V. Studer, A. Pepin, Y. Chen, and A. Ajdari, Analyst (Cambridge, U.K.) 129, 944 (2004).

[12] B. P. Cahill, L. J. Heyderman, J. Gobrecht, and A. Stemmer, Phys. Rev. E 70, 036305 (2004).

[13] A. Ramos, H. Morgan, N. G. Green, A. Gonzalez, and A. Castellanos, J. Appl. Phys. 97, 084906 (2005).

[14] P. Garcia, A. Ramos, N. G. Green, and H. Morgan (unpublished).

[15] A. Ramos, A. Gonzalez, A. Castellanos, N. G. Green, and H. Morgan, Phys. Rev. E 67, 056302 (2003).

[16] D. Lastochkin, R. Zhou, P. Wang, Y. Ben, and H.-C. Chang, J. Appl. Phys. 96, 1730 (2004).

[17] R. J. Hunter, Foundations of Colloid Science (Oxford University Press, New York, 1991).

[18] A. Bard and L. Faulkner, Electrochemical Methods (Wiley, New York, 1980).

[19] M. Z. Bazant, K. Thornton, and A. Ajdari, Phys. Rev. E 70, 021506 (2004).

[20] N. A. Mortensen, L. H. Olesen, L. Belmon, and H. Bruus, Phys. Rev. E 71, 056306 (2005).

[21] I. Rubinstein and B. Zaltzman, Math. Models Meth. Appl. Sci. 11, 263 (2001).
[22] L. H. Olesen (unpublished), following an approach initially suggested in Y. Ben (unpublished).

[23] F. Nadal, F. Argoul, P. Krestener, B. Pouligny, C. Ybert, and A. Ajdari, Eur. Phys. J. E 9, 387-399 (2002).

[24] Ramos et al., used the width $W_{2}$ of the wide electrode as their characteristic length scale rather than $G_{1}$. Hence in [15] both the dimensionless frequency and slip velocity are larger than ours by a factor of $W_{2} / G_{1}$.

[25] A well-known instance of this singular behavior is that at high frequency, where the screening is negligible, the potential close to the sharp electrode edge goes like $\phi \sim r^{1 / 2} \cos (\theta / 2)$, and the electric field diverges as $|\nabla \phi| \sim r^{-1 / 2}$. Here $r$ is the distance from the edge and $\theta$ the polar angle. At finite frequency the normal field is linked to the potential drop across the double layer through Eq. (25) and therefore cannot be singular. However, the tangential field diverges logarithmically. In particular, at low frequency it can be shown analytically that the potential to first order in $\omega$ goes like $\phi$ $\sim \operatorname{Im}\left[\operatorname{Li}_{2}\left(e^{-2 \pi\left(i\left(x-x_{j}\right)+y\right) / L}\right)\right]$ close to the electrode edge [22] and the tangential field diverges as $\left|\partial_{x} \phi\right| \sim-\log \left|x-x_{j}\right|$. Here $\operatorname{Li}_{2}(z)=\sum_{n=1}^{\infty} z^{n} / n^{2}$ is the dilogarithm function.

[26] At high frequency $\tilde{u}_{s}$ is nonzero only in a region of width $\widetilde{\omega}^{-1}$ from the electrode edges. Therefore our numerical solution becomes inaccurate for $\widetilde{\omega} \gtrsim 10^{3}$ and likewise for $K \gtrsim 10^{3}$ in the case of Faradaic current injection.

[27] A. Ajdari, C. R. Phys. 5, 539 (2004).

[28] Experimentally, it is often found even for perfectly polarizable solid electrodes that the double-layer impedance does not match that of a perfect capacitor [29]. Some authors therefore find it efficient to use the empirical so-called constant-phase element to model the double layer, but we prefer not to do so due to the lack of structural justification for it.

[29] Z. Kerner and T. Pajkossy, Electrochim. Acta 46, 207211 (2000).

[30] W. H. Smyrl and J. Newman, Trans. Faraday Soc. 63, 207 (1967).

[31] K. T. Chu and M. Z. Bazant, SIAM J. Appl. Math. 65, 1485 (2005).

[32] P. J. Sides, Langmuir 19, 2745 (2003).

[33] J. A. Fagan, P. J. Sides, and D. C. Prieve, Langmuir 21, 1784 (2005). 